\title{
A Decision Support System for Wind Power Production
}

\author{
Ruggero Donida Labati, Member, IEEE, Angelo Genovese, Member, IEEE, \\ Vincenzo Piuri, Fellow, IEEE, Fabio Scotti, Senior Member, IEEE, and Gianluca Sforza, Member, IEEE
}

\begin{abstract}
Renewable energy production is constantly growing worldwide, and some countries produce a relevant percentage of their daily electricity consumption through wind energy. Therefore, decision support systems that can make accurate predictions of wind-based power production are of paramount importance for traders operating in the energy market and for managers in charge of planning the non-renewable energy production. In this paper, we present a decision support system that can predict electric power production, estimate a variability index for the prediction, and analyze wind farm production characteristics. The main contribution of this paper is a novel system for long-term electric power prediction based solely on weather forecasts; thus, it is suitable for wind farms that cannot collect or manage real-time data acquired by sensors. Our system is based on neural networks and on novel techniques for calibrating and thresholding the weather forecasts based on the distinctive characteristics of wind farm orography. We tuned and evaluated the proposed system using data collected from two wind farms over a two-year period and achieved satisfactory results. We studied different feature sets, training strategies, and system configurations before implementing this system for a player in the energy market. This company evaluated the power production prediction performance and impact of our system at ten different wind farms under real-world conditions and achieved a significant improvement with respect to their previous approach.
\end{abstract}

Index Terms-Renewable energy, Wind energy, Prediction, Calibration, Orography, Neural Networks, Decision support system.

\section{INTRODUCTION}

$\mathbf{T}$ HE importance of green technologies and renewable energy sources is constantly increasing [1]. In this scenario, the research community is studying novel energy efficient production strategies [2], [3], efficient buildings [4], smart grid technologies [5], and techniques for predicting electric loads [6] to determine the optimal price of renewable energy [7]. Among these topics, increasing renewable energy production and improving distribution are of paramount importance to the global economy.

Wind is one of the most important, sustainable, and ecological sources of renewable energy [8]. A market analysis [9] estimates that wind farm energy production is growing at $16.1 \%$ per year and currently meets approximately $5 \%$ of global electricity consumption.

Ruggero Donida Labati, Angelo Genovese, Vincenzo Piuri, and Fabio Scotti are with the Department of Computer Science, Università degli Studi di

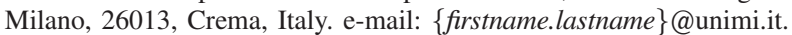

Gianluca Sforza is with the Istituto Nazionale di Fisica Nucleare, Bari Section, 70126 Bari, Italy. e-mail: gianluca.sforza@ba.infn.it
Efficiently managing energy production in a geographical region requires estimating the amount of produced renewable energy so that non-renewable power production can be planned accordingly to satisfy the overall energy need [10]. However, the market for renewable energy penalizes producers when their estimated power production differs from that actually delivered [11]. To avoid penalties and maximize their income, companies need accurate power predictions and decision support systems [4], [12], [13].

In ideal conditions, wind power production is defined as a physical relationship between the wind speed, density of air, and swept area of the turbine [14], [15]. However, even if a direct relation truly existed between wind speed and the power generated by the turbines in wind farms, wind energy production is a dynamic and non-linear process that is affected by several difficult-to-predict aspects [16], such as the $i$ ) orography of the territory [17], ii) turbine positions [18], iii) windmill blade orientation, and $i v$ ) other turbine technical features. For example, the relative locations of the turbines can create shadowing (or wake) effects [19]; offshore and nearshore wind farms can be affected by periodic large wind speed fluctuations [20], and wind farms built close to hills can be influenced by terrain irregularities (Fig. 1).

In many application scenarios, the only available information for predicting power production is the Numerical Weather Prediction (NWP), which is always affected by uncertainty due to the time interval elapsed between its computation and the predicted instant of power generation [21]. Furthermore, the geographical coordinates related to NWP data do not usually correspond to the virtual center of the wind farm because NWP are computed for grids of equally-spaced points [22].

Most power prediction methods in the literature are based on NWP or on historical power prediction data [23], [24]. Depending on the considered prediction horizon, these methods can predict power production at intervals ranging from extremely short-term (a few seconds) to relatively long-term (one day to one week). Most works address short-time predictions (from approximately 30 minutes to 6 hours ahead) [14]. The approaches for long-term predictions can be divided into physical, statistical and hybrid techniques [25].

Physical techniques compute the vertical wind profile at the wind farm to correct the forecasted wind speed by considering several factors, including the distance of the actual plant from the NWP grid, site orography, and turbine height with respect to the NWP reference height [26], [27]. Statistical techniques use time series of the measured power and historical meteorological data [24]. Hybrid techniques use all the available 
(a)

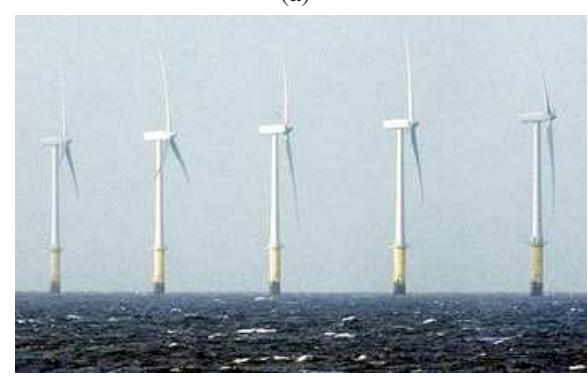

(b)

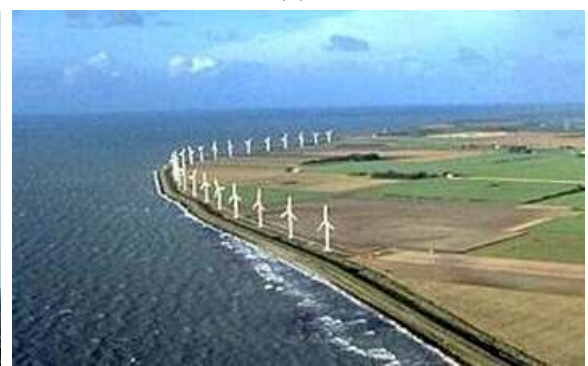

(c)

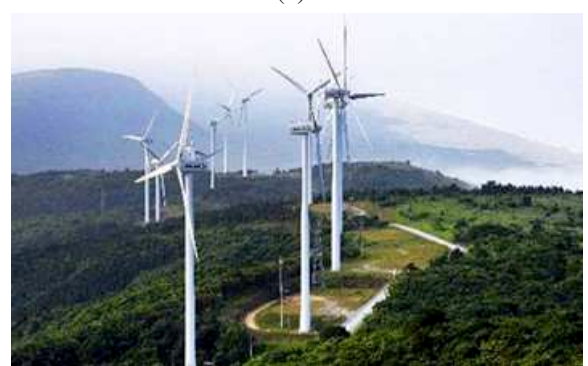

Fig. 1. Different wind farm environmental conditions profoundly influence the production and the complexity of the power prediction for (a) offshore wind farms, (b) nearshore wind farms affected by periodic breezes, and (c) wind farms with complex orography and nearby hills.

information and are often based on computational intelligence techniques [28].

It has been proved that physical models do not provide satisfactory accuracy due to the high complexities and nonlinearities involved in wind power prediction. Statistical and hybrid techniques usually obtain better performance, and hybrid techniques are the most effective for a wide set of application scenarios. However, most of the hybrid methods in the literature require real-time data acquired by wind farm sensors (which may not be available for some application scenarios), do not consider the uncertainty in NWP data stemming from temporal and geographical factors, and do not exploit information on the orography and production characteristics of the wind farm.

The motivations of this work are to propose a new power prediction approach able to overcome those limitations of the current hybrid techniques and present novel decision support tools for end-users working in the energy market.

We propose a novel decision support system that provides information on the power produced by a single wind farm. The system includes a hybrid method based on computational intelligence for long-term power prediction, a function to estimate a variability index of the predicted power, and tools for analyzing wind farm production characteristics. Compared to other methods in the literature, the advantages of our system are as follows: it is based solely on NWP data and can therefore be used for every type of wind farm; it reduces NWP uncertainty by automatically learning and exploiting information about the orography of the territory via the productive characteristics of the wind farm (no GIS or mapping information are required); and it automatically learns the wind farm characteristics using a novel technique for calibrating wind forecasts and a novel algorithm for thresholding the wind forecasts.

To predict power production, we exploit sets of neural networks in conjunction with the wind calibration and thresholding modules. We use NWP related to different points of the weather forecast grid obtained at different times. We also consider heterogeneous training strategies, configurations of the calibration and wind thresholding modules, and numbers of neural predictors.

As a case study, we considered the day-after market [11], but our system is general and can be applied to different scenarios. We performed most of the experiments using data related to two wind farms that cover a two-year period and achieved satisfactory results. Subsequently, a player in the energy market adopted our decision support system and evaluated its performance on ten other wind farms, achieving a significant improvement with respect to their previous system.

The remainder of this paper is structured as follows. Section II describes related works for long-term wind-based power production predictions. Section III presents the system to estimate power production and the related neural prediction variability index. Section IV describes the decision support tools designed to analyze wind farm production characteristics. Section V presents the results obtained using a real-world case study, including the selected metrics, system configurations tested, and the validation methods. Finally, Section VI concludes this work.

\section{RELATED WORKS}

Several methods in the literature have used computational intelligence techniques for environmental monitoring [29][31], time series prediction [32], [33], prediction of adverse environmental conditions [34], and renewable energy forecasting [35]-[37] because such techniques adapt well to noisy data and variations in operational conditions.

In the field of wind power forecasting, studies have shown that computational intelligence techniques usually outperform methods based on auto-regressive models, especially in cases where training datasets with large numbers of samples are available [14], [38], [39].

Many methods for predicting power production have also applied computational intelligence techniques such as artificial neural networks, deep learning, support vector machines, or fuzzy rules to aggregate historical data of predicted power [40]-[43]. Several approaches have used neural networks and evolutionary computation to aggregate the output of different predictors [40], [44]. In addition, some methods have considered neural networks [45] and fuzzy systems [16], [46] for wind farm design and control. In several cases, evolutionary computation has been used in combination with neural networks to optimize the learning process [47]-[50]. The prediction accuracy of such methods depends on the learning method used, the accuracy of the input data [51], [52], and the advance period of the required predictions [24].

Frequently used features include historical meteorological data [23], [24], [53], spatial information [54], real time mea- 
surements performed using wind farm sensors [55], and NWP [25] data.

However, in many application scenarios, NWP data are the only available information for predicting power production: power production data may be available only after a delay; historical data may cover insufficient time periods; spatial information may be limited or incomplete; and collecting realtime measurements from sensors placed in the wind farm is not always possible, particularly for wind farms located far from cities. However, NWP data present inaccuracies due to the specific orography of the wind farm and to differences between the spatial coordinates for which they have been computed and the coordinates of the windblades. Furthermore, large wind farms can include coordinates from multiple points of the weather forecast grid, and small plants may be completely included in a grid rectangle [51].

To compensate for the NWP data inaccuracies, some methods in the literature have estimated the quality of the weather forecasts [56], [57], evaluated the forecasting error [21], [38], [58], [59], performed preliminary feature selection [49], [60], or enhanced the NWP data by considering mesoscale models as the source of weather forecasts [28], [61], [62]. Other approaches have integrated the NWP data with local observations [63], terrain data, and orography information to downscale the NWP forecasts to a smaller areas (e.g., an area of $1 \mathrm{~km} \times 1 \mathrm{~km}$ ). Examples of mesoscale models include the Fifth-Generation Mesoscale Model (MM5) [64], the Weather Research \& Forecasting Model (WRF) [65], and the Aire Limitée Adaptation dynamique Développement InterNational (ALADIN) [66].

To downscale NWP data to smaller areas, the method proposed in [58] first uses the WRF and ALADIN mesoscale models and then analyzes the correlation between the NWP data and the power generated by each turbine. Next, this method clusters the obtained forecasts and historical generated power data to highlight similar patterns. It finally predicts production 2 days ahead using a combination of neural networks and support vector machines. Similarly, the method described in [21] improves the NWP by performing a WRF simulation and cluster analysis to search for correspondences between forecasting errors and NWP values, while [38] uses an error-correcting model for NWP that analyzes the differences between the weather forecasts and actual wind speed measurements. The method proposed in [59] adopts the ALADIN mesoscale models and uses polynomial neural networks to improve the NWP for a specific site. However, these methods require real-time input data collected by sensors placed in the wind farm. Thus, they are not applicable to many wind farms.

The literature does contain prediction methods designed for application scenarios similar to the one considered in this paper. The methods described in [28], [61], [62] use neural networks and the MM5 model to predict the wind power generated by each turbine of the power plant two days ahead. However, these methods require real-time measurements and require information on the local orography and terrain. In contrast, our system infers the orography information from the data; thus, it is both simpler and more suitable for a wider range of application scenarios. The methods described in [56],
[57] predict the power two days in advance using only NWP data. These studies use self-organizing maps to cluster the wind data according to the speed and then processed each cluster using a radial basis function neural network. Finally, these methods refine the obtained prediction using fuzzy logic techniques. The parameters of the fuzzy techniques should be tuned by a human expert for each wind farm separately. In contrast, our system requires no advance knowledge about the wind farm because it automatically adapts to heterogeneous application conditions. The method described in [67] uses a k-means clustering algorithm to divide the NWP data; then, each cluster is used to train a neural network that performs predictions. The clustering method increases the accuracy of the neural predictor by simplifying its input data but, differently from our power prediction approach, it does not compensate for NWP data inaccuracies according to the characteristics of a specific wind farm.

\section{THE PROPOSED SYSTEM FOR LONG-TERM POWER PREDICTION AND ESTIMATION OF THE NEURAL PREDICTION VARIABILITY INDEX}

We propose a novel system for long-term power prediction that automatically learns the orography information and the wind farm characteristics from the NWP data. This system also estimates a neural prediction variability index for the power prediction. The system is designed to be used by traders working in the energy market and by managers who need to plan the production of non-renewable energy.

Fig. 2 shows the schema of our system for predicting power production and estimating the corresponding neural prediction variability index. The system includes two main blocks: preprocessing and configuration.

The preprocessing block includes the data harmonization and the feature extraction modules. The first module transforms data collected from different sources into a common measurement system. The second module computes different sets of numerical features from NWP data, based on wind farm characteristics and the available data.

The configuration block is composed of the calibration, thresholding and neural prediction modules. We present six system configurations obtained by rearranging the configuration block modules. The calibration module automatically learns information from the orography and wind farm characteristics and uses them to improve the wind forecasts of NWP data. The thresholding module detects cases in which the wind energy is insufficient to activate the wind farm turbines. The neural prediction module is composed of a hierarchical set of artificial neural networks trained using different strategies.

We estimate the neural prediction variability index of the power prediction by computing statistics from the results of the neural networks that compose the neural prediction module.

We also propose different training strategies for our system based on the considered scenario.

In the following, we present a formal definition of the problem, describe our system for predicting power production and estimating the neural prediction variability index, and describe different training strategies in detail. 


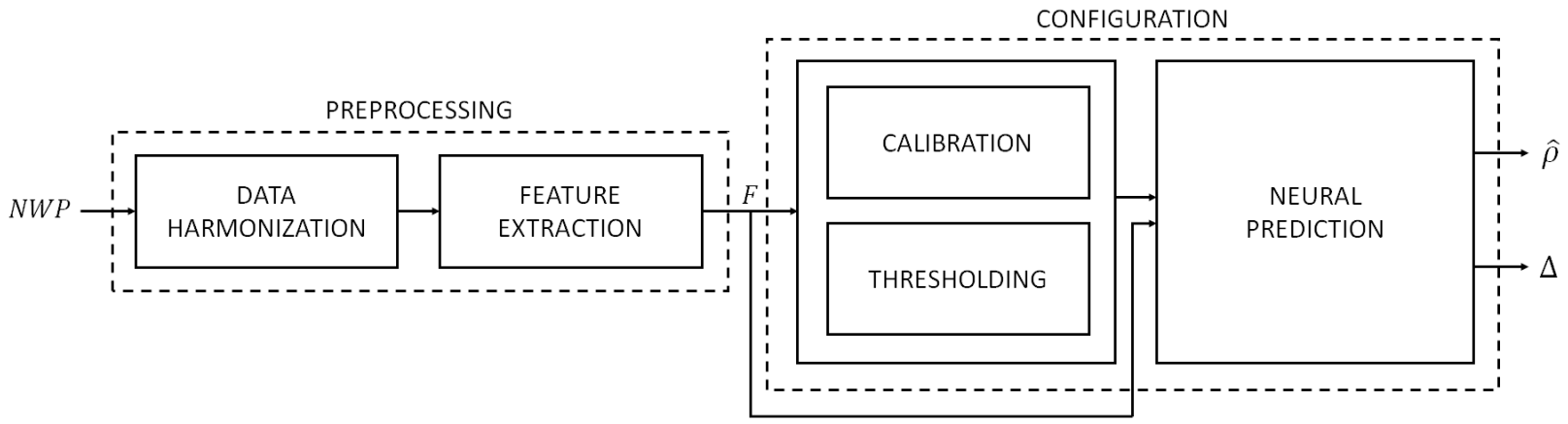

Fig. 2. Schema of the proposed system for predicting power production $\hat{\rho}$ and estimating the neural prediction variability index $\Delta$ of the power prediction. The inputs of the system consist solely of Numerical Weather Predictions (NWP). The calibration and thresholding modules can be used in different configurations according to the target system.

\section{A. Problem definition}

Let $t_{0}$ be the times at which weather forecast data are available, and let NWP be the set of harmonized weather forecasts (more details will be provided in Subsection III-B). Let $\mathcal{T}$ be the ordered set of $n$ times $t_{i} \in \mathcal{T}$ (expressed in hours) for which the weather forecasts are provided. Thus, $\mathcal{T}=\left\{t_{i}\right.$ : $\left.t_{i}<t_{i+1}, i=(1, \ldots, n)\right\}$ and its cardinality is $|\mathcal{T}|=n$. For the considered case study, our system performs predictions for the next day, at intervals of 3 hours, with a time horizon of one day (i.e., where $\mathcal{T}=\left\{t_{0}+24, t_{0}+27, \ldots, t_{0}+45\right\}$, and $n=8$ ). The array of meteorological data available for a given point of the grid $s_{j}$ and forecasted at a certain time $t_{i}$ are defined as follows:

$$
\mathrm{NWP}_{t_{i}, s_{j}}=\left[u_{t_{i}, s_{j}}, v_{t_{i}, s_{j}}, \operatorname{temp}_{t_{i}, s_{j}}, \operatorname{press}_{t_{i}, s_{j}}, \operatorname{cloud}_{t_{i}, s_{j}}\right]
$$

where:

- $u_{t_{i}, s_{j}}$ and $v_{t_{i}, s_{j}}$ represent the wind speed forecasts expressed using two variables for the eastward $\left(u_{t_{i}, s_{j}}\right)$ and northward $\left(v_{t_{i}, s_{j}}\right)$ directions, respectively,

- temp $_{t_{i}, s_{j}}$ is the temperature;

- press $_{t_{i}, s_{j}}$ is the atmospheric pressure; and

- $\operatorname{cloud}_{t_{i}, s_{j}}$ is the cloud coverage.

The generic set of features $F_{t_{i}}$ selected for predicting the power produced at time $t_{i}$, is defined by aggregating NWP arrays processed at different times for different points of the grid. For example:

$$
F_{t_{i}}=\left[\mathrm{NWP}_{t_{i}, s_{1}}^{t_{0}}, \mathrm{NWP}_{t_{i}, s_{2}}^{t_{0}}, \text { date }_{t_{i}}, \text { hour }_{t_{i}}, t_{i}\right],
$$

or

$$
F_{t_{i}}=\left[\mathrm{NWP}_{t_{i}, s_{1}}^{t_{0}}, \mathrm{NWP}_{t_{i}, s_{1}}^{t_{0}-24}, \text { date }_{t_{i}}, \text { hour }_{t_{i}}, t_{i}\right],
$$

where date $t_{i}$ is a numerical vector composed as $\left[\right.$ day $_{t_{i}}$, month $_{t_{i}}$, year $\left._{t_{i}}\right]$ and hour $_{t_{i}}$ is expressed as an integer number (hour $_{t_{i}} \in[0, \ldots, 23]$ ).

Eq. 2 aggregates the NWP data for two sites obtained at time $t_{0}$, while eq. 3 aggregates the NWP data for one site obtained at times $t_{0}$ and $t_{0}-24$.

In the remainder of this paper, we assume that NWPs are obtained at $t_{0}$ unless otherwise indicated (in superscript).
In the simplest configuration of our system, the prediction of wind power at time $t_{i}$ is defined as follows:

$$
\hat{p}_{t_{i}}=\operatorname{PRED}\left(F_{t_{i}}\right),
$$

where $\operatorname{PRED}(\cdot)$ is a power production predictor.

\section{B. Preprocessing}

The preprocessing module converts weather forecast data to a reference measurement system. Then, it computes a feature set $F$ using discriminative values of NWP data related to different geographical coordinates and computed for different times.

1) Data harmonization: The weather forecast data provided by different vendors and data collected by wind farm sensors could be expressed using different measurement units and reference systems. Moreover, subscribed weather forecast services may change over time, causing the provided data to have different measurement units, grid sizes $d$, and be measured at different heights above the ground. The measurement instruments can also be superseded by different models. Therefore, a common reference system should be adopted.

In our system, we use: ${ }^{\circ} \mathrm{C}$ for the temperature, $\mathrm{m} / \mathrm{s}$ for the wind speed in the eastward and northward directions, $\mathrm{hPa}$ for atmospheric pressure, and a scale from 0 to 1 for cloud coverage. Through experiments, we observed that describing the wind speed in the eastward and northward directions allows the neural networks to better learn the prediction problem with respect to polar coordinates. This probably occurs because Cartesian representations do not present any phase jump.

2) Feature extraction: We propose a system that can work with different combinations of features according to the characteristics of an individual wind farm. We create the feature sets using harmonized NWP data related to different points of the weather forecast grid and taken at different times. Eq. 2 and Eq. 3 show examples of these feature set combinations. Section V-C2 provides more details on the considered combinations.

\section{System configuration}

The configuration block is composed of three modules: calibration, thresholding, and a neural predictor. The first 
module adaptively refines the $u_{t_{i}}$ and $v_{t_{i}}$ components of the NWP data to simulate wind forecasts related to the virtual center of the wind farm. The thresholding module evaluates whether the $u_{t_{i}}$ and $v_{t_{i}}$ components of NWP are sufficient to activate the wind farm turbines and produce energy. The neural predictor is composed of multiple neural networks that estimate the power production and the related neural prediction variability index $\Delta_{t_{i}}$.

Because wind farms have different characteristics, we propose different configurations of our system that should be selected based on the application conditions:

- Conf-A: this configuration predicts power production by exploiting only the neural predictor.

- Conf-B: this configuration applies the calibration module before performing neural predictions.

- Conf-C: this configuration applies the thresholding module to $u_{t_{i}}$ and $v_{t_{i}}$ before performing neural predictions. If the wind module $\rho_{t_{i}}=\sqrt{\left(u_{t_{i}}\right)^{2}+\left(v_{t_{i}}\right)^{2}}$ is less than the value $\rho_{t h}$ related to the thresholding modules, the power prediction $\hat{p}_{t_{i}}$ is set to $0 \mathrm{MW}$.

- Conf-D: this configuration applies the calibration and thresholding modules before performing neural predictions. The input to the thresholding module consists of the calibrated wind speeds $u_{t_{i}}^{N N}$ and $v_{t_{i}}^{N N}$. If the wind module $\rho_{t_{i}}^{N N}=\sqrt{\left(u_{t_{i}}^{N} N\right)^{2}+\left(v_{t_{i}}^{N} N\right)^{2}}$ is below the value $\rho_{t h}^{N N}$ from the thresholding modules, the power prediction $\hat{p}_{t_{i}}$ is set to $0 \mathrm{MW}$.

- Conf-E: Similar to Conf-C, this configuration applies the thresholding module before performing neural predictions, but the neural predictor is trained without considering the times $t_{k}$ at which $\rho_{t_{k}}<\rho_{t h}$.

- Conf-F: Similar to Conf-D, this configuration applies the calibration and thresholding modules before performing neural predictions, but the neural predictor is trained without considering the times $t_{k}$ at which $\rho_{t_{k}}<\rho_{t h}$.

Fig. 3 shows the schemas of the proposed configurations. Each module is described below.

1) Calibration: The goal of the calibration module is to estimate the wind in the virtual center $c$ of the wind farm (Fig. 4) using forecasted wind speed data $u_{t_{i}}$ and $v_{t_{i}}$ pertaining to the feature set $F_{t_{i}}$. Real historical values of $u_{t_{i}}^{c}$ and $v_{t_{i}}^{c}$ measured by an anemometer placed in $c$ are frequently available for a period and can therefore be used to design and tune the calibration module. However, these values cannot be accessed in real time at many wind farms; therefore, they cannot be used as features for our neural predictor.

Our calibration method can be defined as follows:

$$
\begin{aligned}
& u_{t_{i}}^{N N}=\operatorname{CALIB}_{u}\left(u_{t_{i}, \text { Near }}, v_{t_{i}, \text { Near }}\right), \\
& v_{t_{i}}^{N N}=\operatorname{CALIB}_{v}\left(u_{t_{i}, \text { Near }}, v_{t_{i}, \text { Near }}\right),
\end{aligned}
$$

where $\operatorname{CALIB}_{u}(\cdot)$ and $\operatorname{CALIB}_{v}(\cdot)$ are two feedforward neural networks, and the wind speed values $u_{t_{i}, \text { Near }}$ and $v_{t_{i}, \text { Near }}$ are related to the weather forecasting grid point nearest to the virtual center $(c)$ of the wind farm.

Each neural network includes a hidden layer composed of an empirically determined number of tan-sigmoidal nodes and an output layer composed of a linear node. The method used for training the neural networks is the Levenberg-Marquardt algorithm. The training step targets are $u_{t_{i}}^{c}$ and $v_{t_{i}}^{c}$.

The calibrated wind speeds are then concatenated to a feature vector $F_{t_{i}}$, as $F_{t_{i}}=\left[F_{t_{i}}, u_{t_{i}}^{N N}, v_{t_{i}}^{N N}\right]$.

The configurations using the calibration module are Conf-B, Conf-D and Conf-F.

2) Thresholding: Turbines produce electricity only when the wind speed in the direction incident to the windmill blades is greater than the minimum threshold value declared by the factory. However, we experimentally observed that wind farms can start producing energy at wind speeds different from the factory-declared minimum threshold value for several reasons. First, the turbines can have different orientations. Second, each turbine of the wind farm may have a different minimum activation speed. Third, some turbines can be damaged or require maintenance.

When applied to NWP data, our technique can be described as follows:

$$
\hat{p}_{t_{i}}=\left\{\begin{array}{cl}
0 & \text { if } \rho_{t_{i}, \text { Near }}<\rho_{t h} \\
\hat{p}_{t_{i}} & \text { otherwise }
\end{array}\right.
$$

where $\rho_{t_{i}, \text { Near }}=\sqrt{\left(u_{t_{i}, \text { Near }}\right)^{2}+\left(v_{t_{i}, \text { Near }}\right)^{2}}$. Similarly, when applied to calibrated data, this technique uses $\rho_{t h}^{N N}$ and $\rho_{t_{i}, N e a r}^{N N}$ (computed from $u_{t_{i}, N e a r}^{N N}$ and $u_{t_{i}, N e a r}^{N N}$ ).

We adaptively estimate the parameters $\rho_{t h}$ and $\rho_{t h}^{N N}$ from a set of training data. We consider the threshold estimation as a binary classification problem. To estimate $\rho_{t h}$, we consider a training set composed of features corresponding to the values $\rho_{t_{k}, \text { Near }}$ for every time $t_{k}$ pertaining to the training interval. We define the two classes based on the actual power production of the wind farm. The positive class is $p_{t_{k}}>0$ and the negative class is $p_{t_{k}}=0$. The value $\rho_{t h}$ is then computed as a threshold corresponding to the equal error rate (EER) of the receiver operating characteristic (ROC) curve (see Fig. 5) [68]. We consider the EER as the working point of the ROC curve because it represents a good tradeoff between the false positive rate (FPR) and the false negative rate (FNR). We estimate $\rho_{t h}^{N N}$ similarly but use a training set composed of features corresponding to the values $\rho_{t_{k}}^{N N}$ for every time $t_{k}$ pertaining to the training interval. Fig. 5 shows an example of the parameter $\rho_{t h}$ selected from the ROC curve.

The configurations Conf-C and Conf-E use the threshold $\rho_{t h}$, while Conf-D and Conf-F uses $\rho_{t h}^{N N}$.

3) Neural predictor: We use a hierarchical regression strategy based on feed-forward neural networks to predict the power production. Because single neural networks may obtain unsatisfactory accuracy for complex problems, many previous studies have used hierarchical approaches based on a pool of regression techniques to achieve higher accuracy [40], [44].

The prediction method used by our system consists of an ensemble of $\mathcal{L}$ networks trained individually. The final power prediction is given by the median value of the results of all the neural networks:

$$
\begin{array}{r}
\hat{p}_{t_{i}}=\operatorname{median}\left[\operatorname{FFNN}_{l}\left(F_{t_{i}}, u_{t_{i}}^{N N}, v_{t_{i}}^{N N}\right)\right], \\
l \in \mathcal{L},
\end{array}
$$

where $\operatorname{FFNN}_{l}(\cdot)$ is a feedforward neural network. 


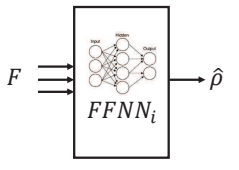

CONF-A

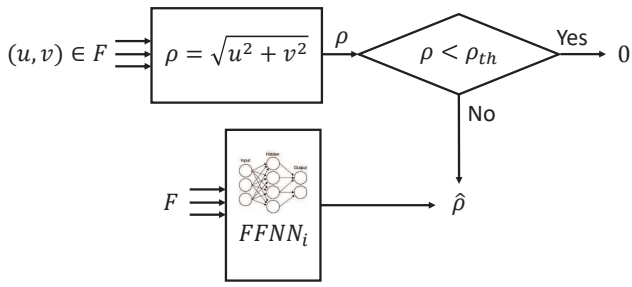

CONF-C/E

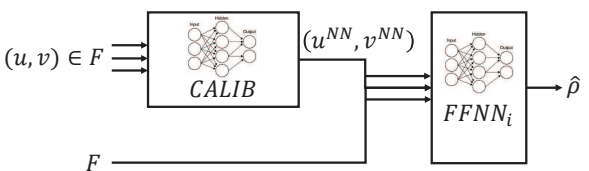

CONF-B

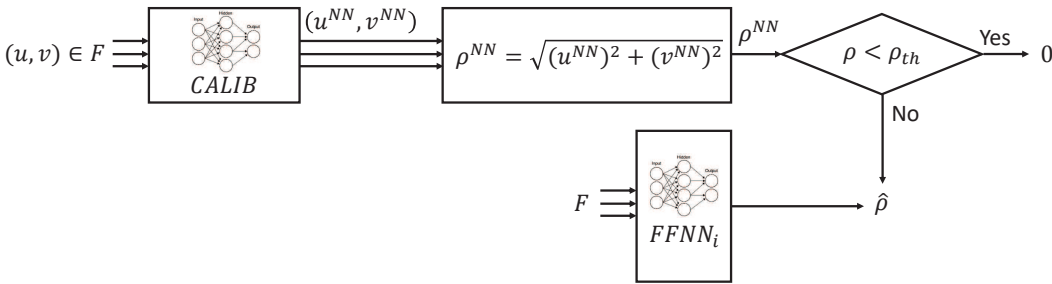

CONF-D/F

Fig. 3. System configurations for long-term power prediction with different combinations of the calibration (CALIB), thresholding, and neural prediction modules based on the considered wind farm characteristics. In the figure, $\rho_{t h}$ is the speed threshold value $\rho=\sqrt{\left(u_{t_{i}}\right)^{2}+\left(v_{t_{i}}\right)^{2}}$ corresponding to the EER; $\rho_{t h}^{N N}$ is similar, but uses the calibrated speed components $u_{t_{i}}^{N N}$ and $v_{t_{i}}^{N N}$. Conf-E differs from Conf-C because the neural prediction module is trained by discarding the samples for times $t_{k}$ at which $\rho_{t_{k}}<\rho_{t h}$. Similarly, Conf-F differs from Conf-D because the neural prediction module is trained using only samples where $\rho_{t_{k}}^{N N}<\rho_{t h}^{N N}$.

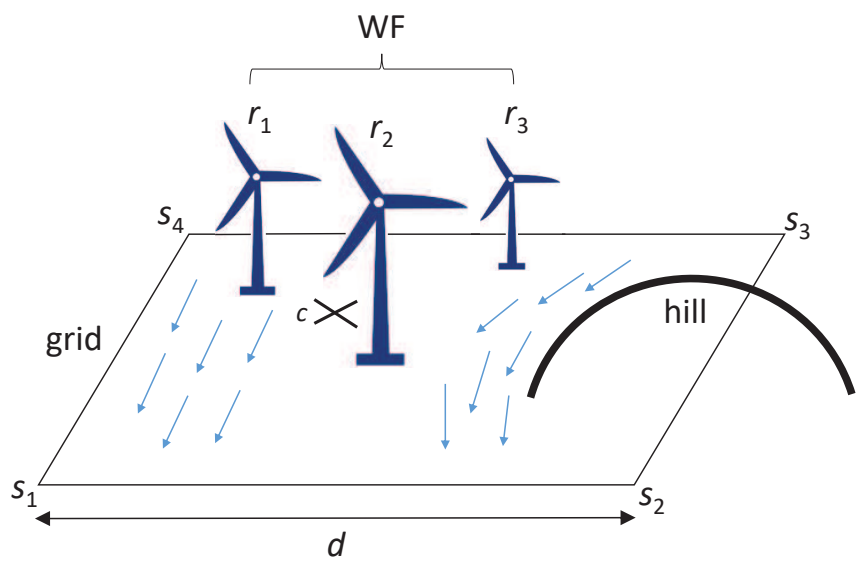

Fig. 4. Example of wind farm (WF) placement in the grid and effect of the orography. The virtual center of the wind farm $c$ composed of different turbines $r_{1}, r_{2}, r_{3}$ is often not horizontally or vertically aligned with the center of the grid. The orography surrounding and within the grid (e.g., the presence of hills) significantly affects wind flow in the wind farm and introduces uncertainty in the weather forecast data, which refers to the coordinates $s_{1}, \ldots, s_{4}$.

Each neural network $\mathrm{FFNN}_{l}$ includes a linear node as an output layer and a single hidden layer composed of an empirically tuned number of tan-sigmoidal nodes. The selected learning technique is based on the Levenberg-Marquardt algorithm.

We also define the neural prediction variability index $\Delta_{t_{i}}$ using the results achieved by the group of neural networks, by considering the inter-percentile range as follows:

$$
\Delta_{t_{i}}=\max \left(m_{t_{i}}^{\beta}-\hat{p}_{t_{i}}, \hat{p}_{t_{i}}-m_{t_{i}}^{\alpha}\right),
$$

where $m_{t_{i}}^{\alpha}$ is the $\alpha$-th percentile and $m_{t_{i}}^{\beta}$ is the $\beta$-th percentile of $l$ predictions, with $\alpha<\beta$.

The model uncertainty estimation can be expressed classically using the variance in the outputs of the multiple neural (a)

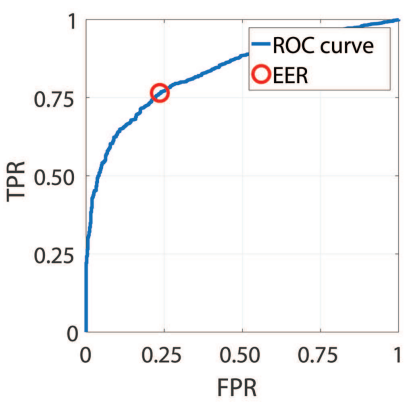

(b)

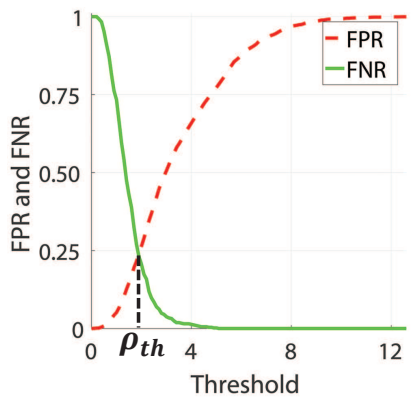

Fig. 5. Example of computing the threshold parameter $\rho_{t h}$ : (a) EER point marked on the ROC curve; (b) FPR and FNR for each possible threshold. The $y$ coordinate of the intersection between the curves FPR and FNR corresponds to the EER, and the $x$ coordinate corresponds to $\rho_{t h}$.

networks, as performed in [40]. However, using this type of application, we cannot make reliable assumptions about the shape and symmetry of the distribution of the neural outputs; thus, we opted for a more pessimistic estimation based on the inter-percentile range, which considers the maximum imbalance among the left and right tails of the distribution.

For our implementation we chose $\alpha$ and $\beta$ values equal to the $10^{\text {th }}$ and $90^{\text {th }}$ percentiles, respectively.

\section{Design of the learning strategy}

To train and validate our system, we consider three strategies:

- $k$-fold cross-validation with random permutations ( $k F$ $R P)$ : this is one of the most commonly used strategies in the literature for building regression and classification models [69]. This strategy has the important advantage of using all the available data for both training and testing a method. For $k$ iterations, kF-RP divides the set of available samples into a validation set composed of 
$1 / k$ of the elements and a training set composed of the remaining samples. The training set is distinct for each iteration and selected to perform a random permutation of the sample indexes. This strategy permits to properly compare the performances of different methods. However, the accuracy values of the time series prediction methods obtained using this strategy do not correspond to the accuracies those methods achieve in real operational conditions because kF-RP does not consider temporal evolution or dependencies in the data [70].

- $k$-fold cross-validation without random permutations ( $k F$ $N O-R P)$ : this strategy is similar to $\mathrm{kF}-\mathrm{RP}$, but the training sets used by the iterative algorithm are obtained by dividing the set of samples into $k$ blocks of fixed size that preserve the temporal order of the samples. This strategy also uses all the available data for both training and validation. Compared to $\mathrm{kF}-\mathrm{RP}, \mathrm{kF}-\mathrm{NO}-\mathrm{RP}$ can reduce problems due to temporal evolution and dependencies in time series.

- Periodic Training (PT): this strategy simulates real operating conditions by periodically training a prediction method. PT divides the available feature set into a training set that includes the samples corresponding to times prior to $t_{s}$ and a validation set composed of the remaining samples. The value of $t_{s}$ is iteratively increased using a constant factor $t_{c}$. Compared to kF-RP and kF-NO-RP, PT does not present problems due to temporal evolution and dependencies in time series because it simulates real operation conditions. However, this strategy allows validating methods over only limited intervals; thus, it reduces the probability that the model will consider all the possible application conditions.

\section{DECISION SUPPORT TOOLS}

In this section, we present two graphical tools intended to provide intelligible information for monitoring the behavior of each individual plant by considering the production and orography of the wind farm.

A physical relationship between wind and power production exists that is based on the following formula [14]: $p_{\text {theory }}=$ $\left(\delta A \rho^{3}\right) / 2$, where $\rho$ is the wind speed $(\mathrm{m} / \mathrm{s}), A$ is the swept area of the turbine $\left(\mathrm{m}^{2}\right), \delta$ is the air density, and $p_{\text {theory }}$ is the expected power (W). However, the above equation does not consider the wind angle. Furthermore, traders and plant engineers are usually interested in aggregating information related to the overall production of the wind farm, where turbines with different orientations and characteristics may exist.

The first tool consists of a set of graphs that evaluate the relationship between the wind angle, its module, and the power produced. This tool permits a visual analysis of the data measured by an anemometer placed in the virtual center of the wind farm $c$. Fig. 6 shows an example of this analysis performed for two wind farms. Fig. 6(a) and Fig. 6(d) show the ratios of power produced to wind speed for discrete sets of wind angles at the two wind farms. Fig. 6(b) and Fig. 6(e) show "Polar boxplots" of the produced power for each set of wind angles at the two wind farms, and Fig. 6(c) and Fig. 6(f) represent the frequency at which the wind angle pertains to each set of wind angles. This figure shows that the two analyzed wind farms exhibit strong differences in terms of orography and production characteristics.

The second tool uses the same types of graphs shown in Fig. 6, but computes them using the NWP data. Comparing the graphs obtained from the anemometer measurements and the NWP data, immediately reveals the accuracy of weather forecasts for a wind farm.

\section{EXPERIMENTAL RESULTS}

This section focuses on the power production prediction performance. First, we present the selected study cases. Second, we describe the metrics used to analyze the power prediction accuracy. Third, we evaluate the performance of the proposed system in different configurations for data related to two heterogeneous wind farms. Finally, we analyze the performance achieved by a player in the energy market using our system in real-world applications and compare the achieved results with that of another commercial system.

\section{A. Study cases}

We initially evaluated the performance of the proposed system using data collected over a two-year period for two different wind farms (WF-A and WF-B). These two wind farms present strong differences in terms of orography. Both wind farms are able to generate a maximum power of approximately $50 \mathrm{MW}$. For each wind farm, we performed power predictions at approximately 3,000 different timepoints.

We used weather forecasting data provided by the European Centre for Medium-Range Weather Forecasts (ECMWF). The forecasting grid used by ECMWF has a scale of $14 \mathrm{~km}$ (i.e., latitude and longitude in 0.125-degree steps) and consists of raw weather forecasting data related to every corner point in the grid.

To study the accuracy of the weather forecasts, we also used data collected by sensors (anemometers) placed at the virtual center $c$ of the considered wind farms. These measurements, however, are affected by noise and instrumental faults, and they are not available for all the predicted times. Furthermore, because this type of data cannot be acquired in real time from all the analyzed power plants, we did not consider these measurements during the design of the proposed system.

\section{B. Metrics}

Power prediction error is commonly computed as $e_{i}=$ $\left(p_{t_{i}}^{*}-\hat{p}_{t_{i}}\right)$, where $p^{*}$ and $\hat{p}$ represent the actual and predicted power production, respectively. Widely used metrics consist of statistical moments computed from the vector $E$ of the prediction errors $e_{i}$.

Other frequently used metrics aim to measure the average error, such as mean absolute error (MAE), its normalized version (NMAE), and root mean square error (RMSE). All these measures are derived from the differences $p_{t_{i}}^{*}-\hat{p}_{t_{i}}$ between the observed and predicted values [71], [72]. MAE is defined as follows: 
(a)
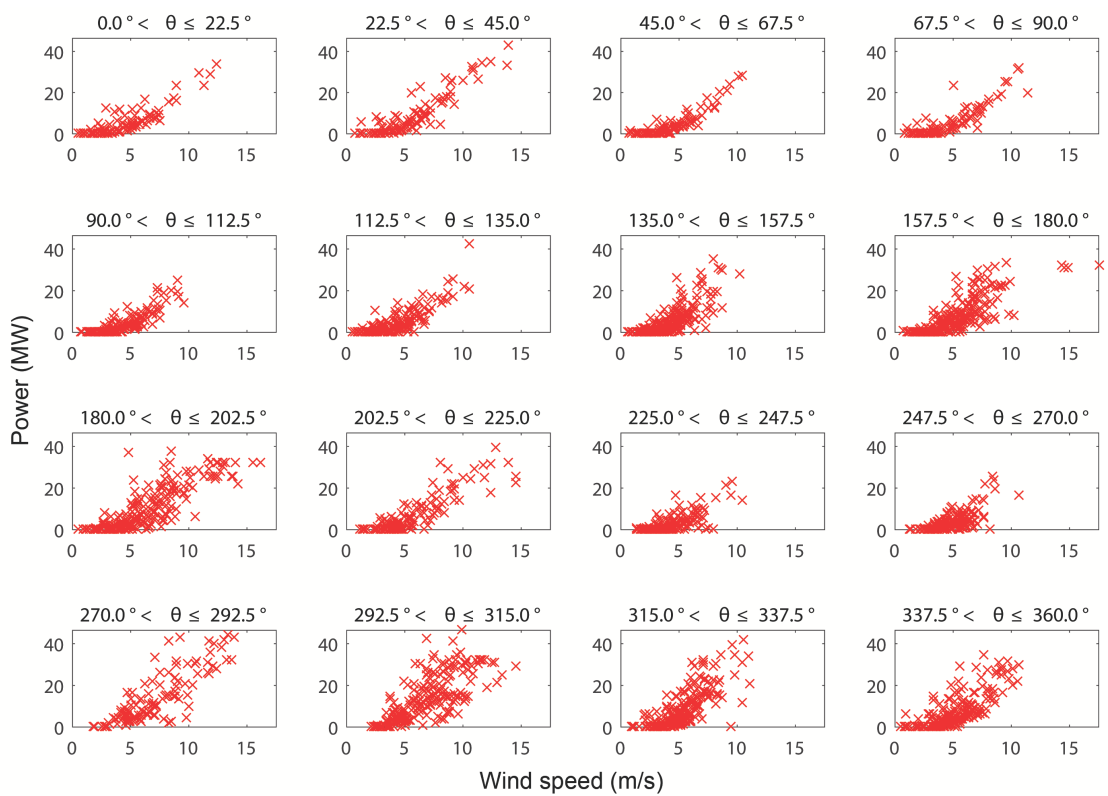

(d)
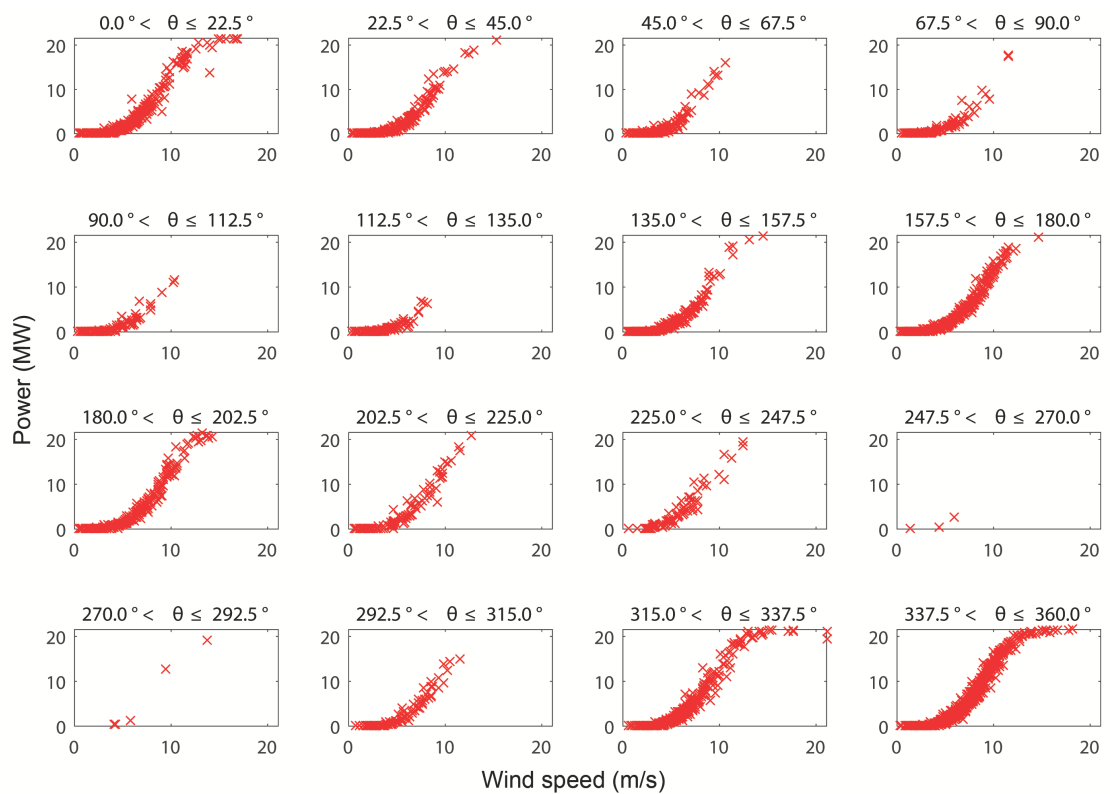

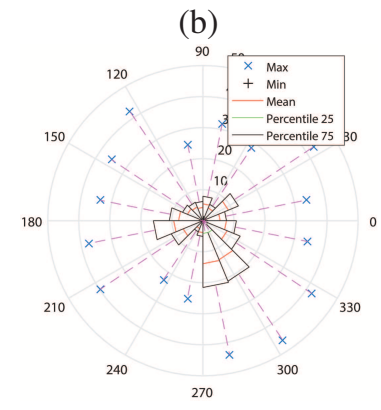

(c)

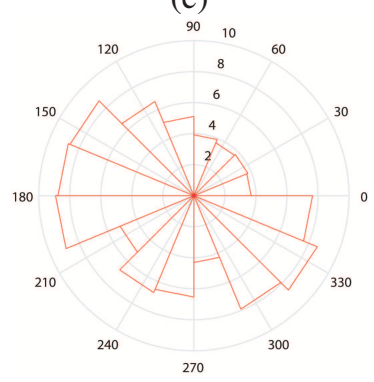

(e)

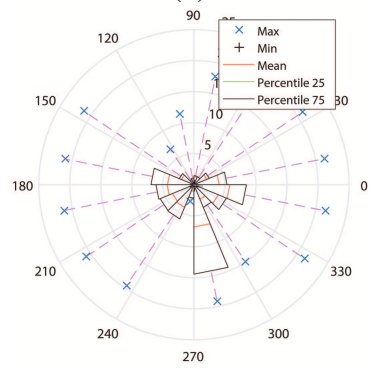

(f)

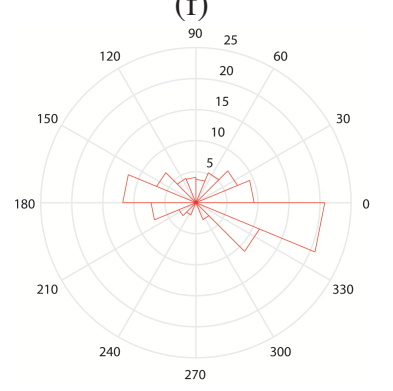

Fig. 6. Proposed graphical decision support tool to evaluate the power production using wind speed measured by an anemometer placed at the virtual center of the wind farm. This tool was applied to wind farms WF-A and WF-B. Images (a), (b) and (c) are related to WF-A, and images (d), (e), (f) are related to WF-B. Images (a) and (d) show the ratios of power produced to wind speed for discrete sets of wind angles. Images (b) and (e) show "Polar box plots" of the produced power for each set of wind angles, and (c) and (f) represent the frequency at which the wind angle pertains to each set of wind angles in (b) and (e), respectively. The variability of the wind speed and the prediction for some angles of WF-A (a) are significantly greater than those of WF-B (d). This compact representation is based on historical data and can help energy producers to verify the neural predictions and make decisions accordingly. The two analyzed wind farms exhibit strong differences in terms of orography and production characteristics.

$$
\mathrm{MAE}=\frac{1}{n} \sum_{i=1}^{n}\left|p_{t_{i}}^{*}-\hat{p}_{t_{i}}\right|
$$

while NMAE and RMSE are respectively defined as follows: NMAE $=\frac{1}{n} \sum_{i=1}^{n}\left(\left|p_{t_{i}}^{*}-\hat{p}_{t_{i}}\right| / c_{p}\right)$, where $c_{p}$ is the net capacity of the plant, and RMSE $=\left[\frac{1}{n} \sum_{i=1}^{n}\left(p_{t_{i}}^{*}-\hat{p}_{t_{i}}\right)^{2}\right]^{1 / 2}$.

An important difference between these metrics lies in their sensitivity to extreme values (outliers) [73]. Metrics such as RMSE are highly influenced by outliers. On the contrary, metrics based on median values are less sensitive to outliers. MAE falls in the middle. The use of measures based on MAE for model comparison is also advantageous because it is a measure of error that can be clearly interpreted [74].

The weighted MAE (WMAE) metric is linked to the stakeholder's cost function, which is defined as follows:

$$
\text { WMAE }=\frac{\sum_{i=1}^{n}\left|p_{t_{i}}^{*}-\hat{p}_{t_{i}}\right|}{\sum_{i=1}^{n}\left|p_{t_{i}}^{*}\right|} .
$$

We used the following metrics: mean error $(\operatorname{Mean}(E))$, 
standard deviation $(\operatorname{Std}(E))$, (Skewness $(E)$ ), quantile 0.1 $(q(E, 0.1))$, median $(q(E, 0.5))$, quantile $0.9(q(E, 0.9))$, MAE, and WMAE.

Within this set of metrics, skewness is important because it reveals the prediction error trend. This metric is negative when power production is underestimated and positive when production is overestimated.

\section{Accuracy evaluation}

In this subsection, we compare the performances of wellknown prediction techniques from the literature, evaluate feature sets composed of weather forecasts related to different points of the grid and predicted two and three days ahead, analyze different training strategies, evaluate the accuracy of different system configurations, and discuss the effect of using pools of neural networks instead of a single regression technique.

1) Power prediction techniques: As starting point for our research, we evaluated the performance of different power prediction techniques from the literature to select the technique that achieves the most promising results.

The persistence of the power produced in previous instants of time is commonly used as a reference for comparing novel models of prediction. The main idea behind this method is to exploit the fact that weather conditions change relatively slowly within the forecasting horizon. It is defined as: $\hat{p}_{t_{i}+\Delta t}=p_{t_{i}}^{*}$, where $p_{t_{i}}^{*}$ is the power measured at time $t_{i}$. We considered the persistence when $\Delta t=48$ hours as Baseline-1.

The second considered method, Baseline-2, computes a constant value, $p$, as the mean of the power produced by the wind farm over the entire test period.

Table I compares the performance of the proposed system in its simplest configuration (Conf-A with a single neural predictor) with that of Baselines 1 and 2 for DB_2_Near (described in Subsection V-C2). We used the kF-RP crossvalidation method with $\mathrm{k}=10$. The results show that the neural approach achieved a higher accuracy with respect to the compared techniques for each of the considered wind farms.

We also considered techniques based on auto-regressive models [75], which achieved unsatisfactory results.

2) Feature set: To tune the proposed system, we searched for the best configuration of the feature set by evaluating relevant combinations of weather forecasts at different spatial coordinates $s_{j}$ (with $1 \leq j \leq 4$ ) and forecasts performed both 2 and 3 days ahead of time $t_{0}$ (with $t_{i} \in\left\{t_{0}+24, t_{0}+\right.$ $\left.\left.27, \ldots, t_{0}+69\right\}\right)$. In this manner, we created 3 feature sets based on the number of sites considered:

1) DB_Near: NWP data related to the point of the grid closest to the considered wind farm. Each sample was composed of the following features: $F_{t_{i}}=$ $\left[N W P_{t_{i}, s_{1}}\right.$, date $_{t_{i}}$, hour $\left._{t_{i}}, t_{i}\right]$, where $s_{1}$ represents the closest site and $t_{i} \in \mathcal{T}$.

2) DB_WMean: weighted mean of NWP data for the four grid sites surrounding the considered wind farm, with weights computed according to the distance between each site and the wind farm. Each sample was composed of the following
10 features: $F_{t_{i}}=\left[\left(N W P_{t_{i}, s_{1}}\left(d_{1} / d_{T o t}\right)+\right.\right.$ $N W P_{t_{i}, s_{2}}\left(d_{2} / d_{\text {Tot }}\right)+W P_{t_{i}, s_{3}}\left(d_{3} / d_{\text {Tot }}\right)+$ $\left.N W P_{t_{i}, s_{4}}\left(d_{4} / d_{\text {Tot }}\right)\right)$, date $t_{i}$, hour $\left._{t_{i}}, t_{i}\right]$, where $\left(d_{1}, d_{2}\right.$, $\left.d_{3}, d_{4}\right)$ are the distances between each point of the grid $s_{i}, d_{T o t}=d_{1}+d_{2}+d_{3}+d_{4}, c$ represents the center of the wind farm, and $t_{i} \in \mathcal{T}$.

3) DB_Corners: NWP samples from the four corners of the rectangular grid. Each sample was composed of the following features: $F_{t_{i}}=\left[N W P_{t_{i}, s_{1}}, N W P_{t_{i}, s_{2}}, N W P_{t_{i}, s_{3}}\right.$, $N W P_{t_{i}, s_{4}}$, date $_{t_{i}}$, hour $\left._{t_{i}}, t_{i}\right]$, with $t_{i} \in \mathcal{T}$.

Each feature set was created in two versions. The first version consisted of two-day-ahead weather forecasts, denoted by the prefix DB_2 (e.g., DB_2_Near). The other version collected both two-day- and three-day-ahead weather forecasts, denoted by the prefix DB_2+3 (e.g., DB_2+3_Near). The motivation for creating the latter type of feature set was to exploit the uncertainty of the weather forecasts as additional information for training the neural predictors.

Table II reports the best results achieved by our system in its simple configuration (Conf-A with a single feedforward neural network) for different sets of features created considering the weather forecasts related to different combinations of points on the forecasting grid. We obtained these results using the kF-RP cross-validation approach with $k=10$. The results show that using all the available information (DB_2_Corners) increases the prediction accuracy. This result probably occurred because neural networks can infer more information about wind farm orography by using the weather forecasts for all the available surrounding points of the grid.

Table III presents the best results achieved using our system in its simplest configuration (Conf-A with a single feedforward neural network) and different datasets of features created considering NWP performed 2 and 3 days ahead of time $t_{0}$. We used kF-RP with $\mathrm{k}=10$. The results show that DB_2+3_Corners achieved the best performance. This result is probably due to the increased capability of the neural networks to infer information concerning weather forecast uncertainty by comparing the values computed at different times.

We considered two different methods to normalize the values of the feature sets: min-max normalization and z-score normalization. However, these normalization algorithms did not contribute satisfactorily to the power prediction accuracy. We also evaluated the effectiveness of various feature selection strategies [76] but obtained no significant performance improvements.

The following tests refer to DB_2+3_Corners.

3) Training strategies: We evaluated three different training strategies to properly analyze the performance of the proposed system: kF-RP, kF-NO-RP, and PT (Section III-D). Specifically, we evaluated kF-RP with $k=10$ and kF-NO-RP with $k=10$ and $k=24$ (in the second case, each fold corresponds to one month of the considered two-year datasets). For PT, we simulated the real use of the system over a time span of five months, training the neural classifier every 14 and 31 days.

Table IV summarizes the best results achieved for the best feature set configuration (DB_2+3_Corners) using ConfA with a single feedforward neural network. These results 
TABLE I

COMPARISON OF OUR POWER PREDICTION SYSTEM (CONF-A WITH A SINGLE NEURAL NETWORK) WITH BASELINE ALGORITHMS FOR DB_2_NEAR, EVALUATED WITH KF-RP $(\mathrm{K}=10)$.

\begin{tabular}{lllccccccc}
\hline \hline $\begin{array}{l}\text { Wind } \\
\text { farm }\end{array}$ & $\begin{array}{l}\text { Prediction } \\
\text { method }\end{array}$ & $\begin{array}{l}\text { Mean }(\boldsymbol{E}) \\
{[\mathbf{M W}]}\end{array}$ & $\begin{array}{l}\text { Std( } \boldsymbol{E}) \\
{[\mathbf{M W}]}\end{array}$ & $\begin{array}{l}\text { Skewness }(\boldsymbol{E}) \\
{[\mathbf{M W}]}\end{array}$ & $\begin{array}{l}\mathbf{q}(\boldsymbol{E}, \mathbf{0 . 1}) \\
{[\mathbf{M W}]}\end{array}$ & $\begin{array}{l}\mathbf{q}(\boldsymbol{E}, \mathbf{0 . 5}) \\
{[\mathbf{M W}]}\end{array}$ & $\begin{array}{l}\text { q(E, } \boldsymbol{0 . 9}) \\
{[\mathbf{M W}]}\end{array}$ & $\begin{array}{l}\text { MAE } \\
{[\mathbf{M W}]}\end{array}$ & WMAE \\
\hline WF-A & Baseline-1 & -0.006 & 11.074 & -0.023 & -12.676 & -0.056 & 12.911 & 7.610 & 1.053 \\
WF-A & Baseline-2 & -3.258 & 8.746 & -1.623 & -16.761 & 0.000 & 3.956 & 6.068 & 0.841 \\
WF-A & Conf-A & -0.013 & 4.759 & -0.575 & -5.492 & 0.246 & 5.258 & $\mathbf{3 . 5 1 4}$ & $\mathbf{0 . 4 8 7}$ \\
\hline WF-B & Baseline-1 & -0.011 & 6.484 & -0.020 & -7.602 & 0.000 & 7.490 & 4.292 & 1.173 \\
WF-B & Baseline-2 & -2.146 & 5.016 & -1.777 & -9.971 & 0.000 & 1.505 & 3.317 & 0.909 \\
WF-B & Conf-A & 0.002 & 2.572 & -0.674 & -3.024 & 0.243 & 2.591 & $\mathbf{1 . 6 9 1}$ & $\mathbf{0 . 4 6 3}$ \\
\hline \hline
\end{tabular}

Notes: Baseline $1=$ persistence with $\Delta t=48$ hours; Baseline $2=$ mean of the power produced by the wind farm over the entire test period; Conf-A $=$ the proposed system in its simplest configuration with a single feedforward neural network. Here, $\mathrm{q}(\cdot)$ represents the quantile operation.

TABLE II

SYSTEM ACCURACY (CONF-A WITH A SINGLE NEURAL NETWORK) USING WEATHER FORECASTS RELATED TO DIFFERENT POINTS OF THE GRID AND $\mathrm{KF}-\mathrm{RP}(\mathrm{K}=10)$

\begin{tabular}{|c|c|c|c|c|c|c|c|c|c|c|}
\hline $\begin{array}{l}\text { Wind } \\
\text { farm }\end{array}$ & Feature set & $\begin{array}{c}\text { Hidden layer } \\
\text { nodes }\end{array}$ & $\begin{array}{l}\operatorname{Mean}(E) \\
{[M W]}\end{array}$ & $\begin{array}{l}\operatorname{Std}(E) \\
{[M W]}\end{array}$ & $\begin{array}{l}\text { Skewness }(E) \\
{[\mathrm{MW}]}\end{array}$ & $\begin{array}{l}\mathbf{q}(E, 0.1) \\
{[\mathrm{MW}]}\end{array}$ & $\begin{array}{l}\mathrm{q}(E, 0.5) \\
{[\mathrm{MW}]}\end{array}$ & $\begin{array}{l}\mathrm{q}(\boldsymbol{E}, \mathbf{0 . 9}) \\
{[\mathrm{MW}]}\end{array}$ & $\begin{array}{l}\text { MAE } \\
{[\text { MW] }}\end{array}$ & WMAE \\
\hline WF-A & DB_2_Near & 40 & -0.013 & 4.759 & -0.575 & -5.492 & 0.246 & 5.258 & 3.514 & 0.487 \\
\hline WF-A & DB_2_WMean & 30 & 0.030 & 4.994 & -0.744 & -5.806 & 0.423 & 5.554 & 3.659 & 0.507 \\
\hline WF-A & DB_2_Corners & 35 & 0.052 & 4.595 & -0.139 & -5.327 & 0.194 & 5.331 & 3.430 & 0.475 \\
\hline WF-B & DB-2-Near & 25 & 0.010 & 2.461 & -0.557 & -2.933 & 0.147 & 2.653 & 2.631 & 0.482 \\
\hline WF-B & DB_2_WMean & 30 & -0.002 & 2.527 & -0.551 & -2.859 & 0.150 & 2.675 & 2.665 & 0.489 \\
\hline WF-B & DB_2_Corners & 5 & 0.002 & 2.572 & -0.674 & -3.024 & 0.243 & 2.591 & 2.526 & 0.463 \\
\hline
\end{tabular}

Notes: DB_2_Near = NWP data related to the point of the grid closest to the considered wind farm; DB_2_WMean = weighted mean of NWP data for the four sites of the grid surrounding the considered wind farm; DB_2_Corners = NWP data from the four corners of the rectangular grid.

TABLE III

SYSTEM ACCURACY (CONF-A WITH A SINGLE NEURAL NETWORK) USING WEATHER FORECASTS PERFORMED AT DIFFERENT TIMES AND KF-RP (K=10)

\begin{tabular}{|c|c|c|c|c|c|c|c|c|c|c|}
\hline $\begin{array}{l}\text { Wind } \\
\text { farm }\end{array}$ & Feature set & $\begin{array}{c}\text { Hidden layer } \\
\text { nodes }\end{array}$ & $\begin{array}{l}\operatorname{Mean}(E) \\
{[M W]}\end{array}$ & $\begin{array}{l}\operatorname{Std}(E) \\
{[M W]}\end{array}$ & $\begin{array}{l}\text { Skewness }(E) \\
{[M W]}\end{array}$ & $\begin{array}{l}\mathrm{q}(E, 0.1) \\
{[\mathrm{MW}]}\end{array}$ & $\begin{array}{l}\mathrm{q}(E, 0.5) \\
{[\mathrm{MW}]}\end{array}$ & $\begin{array}{l}\mathrm{q}(E, 0.9) \\
{[\mathrm{MW}]}\end{array}$ & $\begin{array}{l}\text { MAE } \\
{[\text { MW] }}\end{array}$ & WMAE \\
\hline WF-A & DB_2_Corners & 35 & 0.052 & 4.595 & -0.139 & -5.327 & 0.194 & 5.331 & 3.430 & 0.475 \\
\hline WF-A & DB_2+3_Corners & 40 & 0.070 & 4.383 & -0.207 & -4.990 & 0.159 & 5.052 & 3.182 & 0.441 \\
\hline WF-B & DB_2_Corners & 5 & 0.002 & 2.572 & -0.674 & -3.024 & 0.243 & 2.591 & 1.691 & 0.463 \\
\hline WF-B & DB_2+3_Corners & 30 & 0.020 & 2.157 & -0.416 & -2.469 & 0.108 & 2.452 & 1.585 & 0.434 \\
\hline
\end{tabular}

Notes: DB_2_Corners $=$ NWP performed two days ahead of time $t_{0} ;$ DB_2+3_Corners $=$ NWP performed two days ahead of time $t_{0}$ and NWP performed three days ahead of time $t_{0}$.

show that training the neural networks frequently increased the prediction accuracy. In fact, PT achieved better results when using the 14-day training interval than when using the 31-day interval. Table IV also confirms our assumption that kF-RP overestimates the system accuracy because the training process can exploit additional knowledge on weather periodicity compared to a real application scenario. kF-NO-RP achieved more realistic estimations and its accuracy was more similar to PT (which simulates the system in real application conditions) but it provides the advantage of considering all the available data when testing our system. Therefore, we chose kF-NO-RP with $k=10$ as the training strategy for the subsequent tests.

4) Configurations of the proposed prediction system: The calibration module of our system should be optimized for each considered wind farm. In the following, we describe the evaluation procedures for the calibration, thresholding, and neural prediction modules in the various proposed configurations.

We evaluated different techniques for calibrating the wind forecasts:

- Sum: least mean square approximation of $u_{t_{i}}^{M}=u_{t_{i}}+\alpha_{u}$ and $v_{t_{i}}^{M}=v_{t_{i}}+\alpha_{v}$

- Multiplication: least mean square approximation $u_{t_{i}}^{M}=$ $u_{t_{i}} \cdot \beta_{u}$ and $v_{t_{i}}^{M}=v_{t_{i}} \cdot \beta_{v}$.

- Polynomial 1: least mean square approximation of the first order polynomial mapping of $u$ to $u^{M}$ and the first order polynomial mapping of $v$ to $v^{M}$.

- Polynomial 2: least mean square approximation of the second order polynomial mapping of $u$ to $u^{M}$ and the first order polynomial mapping of $v$ to $v^{M}$.

- RANSAC: approximation based on RANdom SAmple Consensus (RANSAC) of the second order polynomial mapping of $u$ to $u^{M}$ and the second order polynomial mapping of $v$ to $v^{M}$. Compared to the least mean square approximation, RANSAC is more robust to noisy data because it iteratively detects and removes outliers during the fitting process. We evaluated functions with different degrees of freedom and achieved the best results using second order polynomials.

- Neural: our calibration method based on neural networks (Subsection III-C).

Table V summarizes the best performance of each con- 
TABLE IV

SYSTEM ACCURACIES FOR POWER PREDICTION (CONF-A WITH A SINGLE NEURAL NETWORK) EVALUATED USING DIFFERENT TRAINING STRATEGIES.

\begin{tabular}{|c|c|c|c|c|c|c|c|c|c|c|}
\hline $\begin{array}{l}\text { Wind } \\
\text { farm }\end{array}$ & $\begin{array}{l}\text { Training } \\
\text { strategy }\end{array}$ & $\begin{array}{l}\text { Hidden layer } \\
\text { nodes }\end{array}$ & $\begin{array}{l}\operatorname{Mean}(E) \\
{[M W]}\end{array}$ & $\begin{array}{l}\operatorname{Std}(E) \\
{[\mathrm{MW}]}\end{array}$ & $\begin{array}{l}\text { Skewness }(E) \\
{[M W]}\end{array}$ & $\begin{array}{l}\mathbf{q}(\boldsymbol{E}, \mathbf{0 . 1}) \\
{[\mathrm{MW}]}\end{array}$ & $\begin{array}{l}\mathrm{q}(E, 0.5) \\
{[\mathrm{MW}]}\end{array}$ & $\begin{array}{l}\mathbf{q}(E, 0.9) \\
{[\mathrm{MW}]}\end{array}$ & $\begin{array}{l}\text { MAE } \\
{[\text { MW] }}\end{array}$ & WMAE \\
\hline WF-A & $\mathrm{kF}-\mathrm{RP}(\mathrm{k}=10)$ & 40 & 0.070 & 4.383 & -0.207 & -4.990 & 0.159 & 5.052 & 3.182 & 0.441 \\
\hline WF-A & kF-NO-RP $(\mathrm{k}=10)$ & 10 & 0.179 & 5.437 & -0.424 & -5.861 & 0.440 & 6.005 & 3.815 & 0.529 \\
\hline WF-A & kF-NO-RP (k=24) & 10 & 0.012 & 4.824 & -0.426 & -5.498 & 0.259 & 5.502 & 3.571 & 0.495 \\
\hline WF-A & PT (31 days) & 3 & 0.410 & 5.376 & -0.447 & -5.989 & 0.851 & 6.362 & 4.040 & 0.535 \\
\hline WF-A & PT (14 days) & 3 & -0.122 & 5.428 & -0.668 & -6.827 & 0.789 & 5.486 & 3.916 & 0.517 \\
\hline WF-B & kF-RP $(k=10)$ & 30 & 0.020 & 2.157 & -0.416 & -2.469 & 0.108 & 2.452 & 1.585 & 0.434 \\
\hline WF-B & kF-NO-RP $(\mathrm{k}=10)$ & 5 & -0.043 & 2.643 & -0.679 & -3.005 & 0.257 & 2.623 & 1.816 & 0.497 \\
\hline WF-B & kF-NO-RP (k=24) & 20 & -0.007 & 2.289 & -0.305 & -2.802 & 0.127 & 2.640 & 1.690 & 0.463 \\
\hline WF-B & PT (31 days) & 3 & 0.247 & 2.604 & -0.508 & -2.872 & 0.419 & 2.946 & 2.121 & 0.533 \\
\hline WF-B & PT (14 days) & 3 & 0.044 & 2.689 & -0.651 & -3.102 & 0.337 & 2.553 & 1.780 & 0.474 \\
\hline
\end{tabular}

Notes: $\mathrm{kF}-\mathrm{RP}=\mathrm{k}$-fold cross validation with random permutations; $\mathrm{kF}-\mathrm{NO}-\mathrm{RP}=\mathrm{k}$-fold cross validation without random permutations; $\mathrm{PT}=$ periodic training over a maximum testing period of five months.

TABLE V

ACCURACIES OF DIFFERENT METHODS FOR CALIBRATING THE WIND FORECASTS VALIDATED USING KF-NO-RP WITH K $=10$

\begin{tabular}{ll|cc|cc}
\hline \hline $\begin{array}{l}\text { Wind } \\
\text { farm }\end{array}$ & $\begin{array}{l}\text { Calibration } \\
\text { method }\end{array}$ & $\begin{array}{c}\mid c \\
\text { mean }\left(E^{u}\right) \\
{[\mathbf{m} / \mathbf{s}]}\end{array}$ & $\begin{array}{c}\mathbf{s t d}\left(E^{u}\right) \\
{[\mathbf{m} / \mathbf{s}]}\end{array}$ & $\begin{array}{c}\boldsymbol{c}^{\boldsymbol{v}} \\
\mathbf{m e a n}\left(E^{v}\right) \\
{[\mathbf{m} / \mathbf{s}]}\end{array}$ & $\begin{array}{c}\mathbf{s t d}\left(E^{v}\right) \\
{[\mathbf{m} / \mathbf{s}]}\end{array}$ \\
\hline WF-A & Sum & 2.77 & 2.21 & 3.34 & 2.82 \\
WF-A & Multiplication & 2.94 & 2.41 & 3.02 & 2.27 \\
WF-A & Polynomial 1 & 2.76 & 2.20 & 2.98 & 2.23 \\
WF-A & Polynomial 2 & 2.75 & 2.22 & 2.90 & 2.22 \\
WF-A & RANSAC & 2.18 & 2.04 & 2.82 & 3.10 \\
WF-A & Neural & $\mathbf{2 . 1 0}$ & $\mathbf{1 . 8 9}$ & $\mathbf{2 . 4 7}$ & $\mathbf{2 . 1 5}$ \\
\hline WF-B & Sum & 2.60 & 1.83 & 3.16 & 2.60 \\
WF-B & Multiplication & 2.14 & 1.72 & 1.70 & 1.48 \\
WF-B & Polynomial 1 & 2.13 & 1.70 & 1.59 & 1.47 \\
WF-B & Polynomial 2 & 2.12 & 1.70 & 1.59 & 1.47 \\
WF-B & RANSAC & 2.09 & 1.68 & 1.91 & 1.76 \\
WF-B & Neural & $\mathbf{2 . 0 4}$ & $\mathbf{1 . 7 5}$ & $\mathbf{1 . 6 0}$ & $\mathbf{1 . 4 3}$ \\
\hline \hline
\end{tabular}

Notes: Sum $=$ least mean square approximation of $u_{M}=u+\alpha_{u}$ and $v_{M}=v+\alpha_{v} ;$ Multiplication = least mean square approximation $u_{M}=u \cdot \beta_{u}$ and $v_{M}=v \cdot \beta_{v}$; Polynomial $1=$ least mean square approximation of the first order polynomial mapping of $u$ to $u_{M}$; Polynomial $2=$ least mean square approximation of the second order polynomial mapping of $u$ to $u_{M}$; RANSAC: approximation based on RANSAC of the second order polynomial mapping of $u$ to $u_{M}$; Neural $=$ our calibration method based on neural networks (as described in Subsection III-C).

sidered technique for $\mathrm{kF}-\mathrm{NO}-\mathrm{RP}$ with $\mathrm{k}=10$. In this test, we used the error metrics $E_{t_{i}}^{u}=\left|u_{t_{i}}^{N N}-u_{t_{i}, s_{j}}\right|$ and $E_{t_{i}}^{v}=$ $\left|v_{t_{i}}^{N N}-v_{t_{i}, s_{j}}\right|$. The results reported for feedforward neural networks refers to networks with 3 tan-sigmoidal nodes in the hidden layer. The obtained results show that the feedforward neural networks achieved the best accuracy for both the considered wind farms. This result probably occurred because it is difficult to model the complex physical configuration of a wind farm using linear approximation techniques. Therefore, nonlinear regression techniques such as neural networks can obtain better performances. Table $\mathrm{V}$ also shows that the calibration module was more accurate for WF-B than for WF-A. This is probably because the NWP data for WF-A are noisier than those for WF-B.

The parameter used by the wind thresholding module should be tuned for each wind farm. The module considers the $u$ and $v$ values extracted from NWP in the Conf-C and Conf-E configurations to compute the threshold $\rho_{t h}$, while it considers $u^{N N}$ and $v^{N N}$ for the Conf-D and Conf-F configurations
TABLE VI

MEAN OF THE BEST THRESHOLDS COMPUTED FOR CALIBRATED AND UNCALIBRATED DATA, VALIDATED USING KF-NO-RP WITH K $=10$.

\begin{tabular}{cc|cc|cc}
\hline \hline \multirow{2}{*}{ Parameter } & Configurations & \multicolumn{2}{|c|}{ WF-A } & \multicolumn{2}{c}{ WF-B } \\
threan & mean & mean & mean \\
t(m/s) & & EER & & $(\mathbf{m} / \mathbf{s})$ & \\
\hline$\rho_{t h}$ & $($ Conf-C, Conf-E) & 1.710 & 0.237 & 2.600 & 0.237 \\
$\rho_{t h}^{N N}$ & $($ Conf-D, Conf-F) & 3.350 & 0.148 & 1.910 & 0.056 \\
\hline \hline
\end{tabular}

to compute the threshold $\rho_{t h}^{N N}$. The mean of the thresholds computed using kF-NO-RP with $k=10$ are listed in Table VI. The results show important differences in terms of the EER obtained when computing $\rho_{t h}$ and $\rho_{t h}^{N N}$. For WF-B, the calibration significantly reduced the prediction error, while it was not effective for WF-A. This result can be justified by the fact that the weather forecasts for WF-A were significantly less accurate than those for WF-B.

The configurations of the proposed system (Conf-A, ..., Conf-F) use the calibration and thresholding modules in different positions of the computational chain. Table VII summarizes the prediction error achieved by performing $\mathrm{kF}$ NO-RP with $k=10$ for each configuration of our system and using a single neural network. The results show that the configuration choice should be guided by the characteristics of each wind farm. For WF-A, we achieved the best errors $\mathrm{MAE}=3.653 \mathrm{MW}$ and WMAE $=0.506$ using Conf-C. For WF-B, we achieved the best errors MAE $=1.602 \mathrm{MW}$ and WMAE $=0.439$ using Conf-D. The calibration and thresholding modules (Conf-B and Conf-C, respectively) increased the accuracy of the basic configuration of our system (Conf-A) for both the considered wind farms. However, Table VII shows that configurations using both the calibration and thresholding modules (Conf-D and Conf-F) achieved satisfactory results only when the weather forecasts were sufficiently accurate to allow calibration to be performed satisfactorily.

5) Pool of neural networks: We evaluated the prediction accuracy by using a hierarchical pool of neural networks and extracting the median value of their predictions, as described in Section III-C3. Table VIII summarizes the results achieved 
TABLE VII

ACCURACY OF DIFFERENT CONFIGURATIONS OF OUR SYSTEM BASED ON A SINGLE FEEDFORWARD NEURAL NETWORK AND VALIDATED USING KF-NO-RP WITH K $=10$

\begin{tabular}{|c|c|c|c|c|c|c|c|c|c|c|}
\hline $\begin{array}{l}\text { Wind } \\
\text { farm }\end{array}$ & Configuration & $\begin{array}{c}\text { Hidden layer } \\
\text { nodes }\end{array}$ & $\begin{array}{l}\operatorname{Mean}(E) \\
{[M W]}\end{array}$ & $\begin{array}{l}\operatorname{Std}(E) \\
{[M W]}\end{array}$ & $\begin{array}{l}\text { Skewness }(E) \\
{[M W]}\end{array}$ & $\begin{array}{l}\mathrm{q}(E, 0.1) \\
{[\mathrm{MW}]}\end{array}$ & $\begin{array}{l}\mathrm{q}(E, 0.5) \\
{[\mathrm{MW}]}\end{array}$ & $\begin{array}{l}\mathrm{q}(E, 0.9) \\
{[\mathrm{MW}]}\end{array}$ & $\begin{array}{l}\text { MAE } \\
{[\text { MW] }}\end{array}$ & WMAE \\
\hline WF-A & Conf-A & 10 & 0.179 & 5.437 & -0.424 & -5.861 & 0.440 & 6.005 & 3.815 & 0.529 \\
\hline WF-A & Conf-B & 10 & 0.223 & 5.396 & -0.696 & -6.088 & 0.810 & 6.246 & 3.747 & 0.519 \\
\hline WF-A & Conf-C & 5 & -0.284 & 5.522 & -0.517 & -6.660 & 0.000 & 5.241 & 3.653 & 0.506 \\
\hline WF-A & Conf-D & 35 & -1.828 & 5.684 & -0.513 & -8.049 & -1.007 & 3.811 & 3.850 & 0.534 \\
\hline WF-A & Conf-E & 25 & -0.085 & 5.174 & -0.288 & -6.197 & 0.000 & 6.021 & 3.652 & 0.506 \\
\hline WF-A & Conf-F & 5 & -1.991 & 6.117 & -0.693 & -9.336 & -0.967 & 4.925 & 4.276 & 0.593 \\
\hline WF-B & Conf-A & 5 & -0.043 & 2.643 & -0.679 & -3.005 & 0.257 & 2.623 & 1.816 & 0.497 \\
\hline WF-B & Conf-B & 15 & -0.033 & 2.483 & -0.322 & -2.948 & 0.073 & 2.670 & 1.774 & 0.486 \\
\hline WF-B & Conf-C & 10 & -0.337 & 2.594 & -0.525 & -3.124 & -0.024 & 2.404 & 1.644 & 0.450 \\
\hline WF-B & Conf-D & 25 & -0.225 & 2.453 & -0.519 & -2.987 & -0.010 & 2.538 & 1.602 & 0.439 \\
\hline WF-B & Conf-E & 30 & -0.213 & 2.515 & -0.471 & -2.956 & -0.014 & 2.734 & 1.654 & 0.453 \\
\hline WF-B & Conf-F & 35 & -0.181 & 2.451 & -0.325 & -2.813 & -0.018 & 2.570 & 1.618 & 0.443 \\
\hline
\end{tabular}

TABLE VIII

ACCURACY OF OUR BEST SYSTEM CONFIGURATION SYSTEM BASED ON A POOL OF 10 FEEDFORWARD NEURAL NETWORKS, VALIDATED USING KF-NO-RP WITH K $=10$.

\begin{tabular}{ll|cc|cc}
\hline \hline \multirow{2}{*}{$\begin{array}{l}\text { Wind } \\
\text { farm }\end{array}$} & Configuration & \multicolumn{3}{|c|}{ WMAE } & \\
& & Ningle & $\begin{array}{c}\text { Pool of 10 } \\
\text { NN }\end{array}$ & $\operatorname{mean}\left(\Delta_{t_{i}}\right)$ & $\operatorname{std}\left(\Delta_{t_{i}}\right)$ \\
\hline WF-A & PT (Conf-C) & 0.506 & 0.434 & 3.950 & 2.581 \\
WF-B & PT (Conf-D) & 0.439 & 0.380 & 2.545 & 6.096 \\
\hline \hline
\end{tabular}

by applying a single neural predictor and a pool of 10 neural networks for the best-performing configurations of our system. We used kF-NO-RP with $k=10$. For each wind farm, the table reports the mean and standard deviation of the neural prediction variability index $\Delta_{t_{i}}$ of the performed predictions (Eq. 8). In our tests, pooling the neural networks increased the repeatability of the tests and outperformed the single neural predictor, reducing the WMAE by approximately 0.06 $(\approx 12 \%)$.

Fig. 7 shows the actual power $p_{t_{i}}^{*}$ and the predicted power $\hat{p}_{t_{i}}$ using a pool of 10 neural networks for our best-performing system configuration (Table VIII) over a 100-day period. The graphs show that, in the vast majority of cases, our system accurately predicted the production peaks.

\section{Application of the proposed system in the energy market}

This subsection discusses the results obtained by applying a first prototype of our prediction system in the real energy market. A player in the energy market used our system for a time span of 70 days on a set of 10 wind farms and summarized the achieved results.

Table IX compares our system with the commercial system previously adopted by the stakeholder. The results apply to our system in its simplest configuration (Conf-A) with a pool of 100 neural networks trained daily. Conf-A was selected mainly because this was a preliminary test for the application of our system. The results show that, in the vast majority of cases, our system outperformed the previously used predictor. (a)

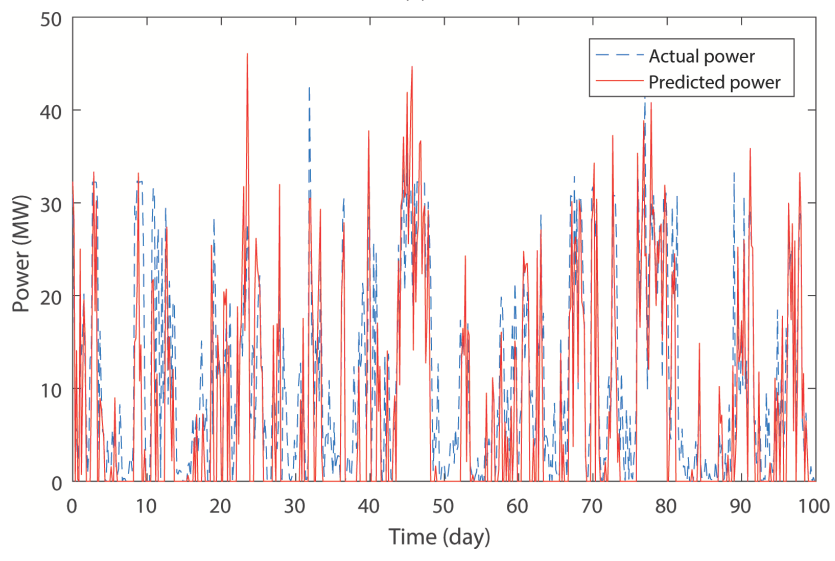

(b)

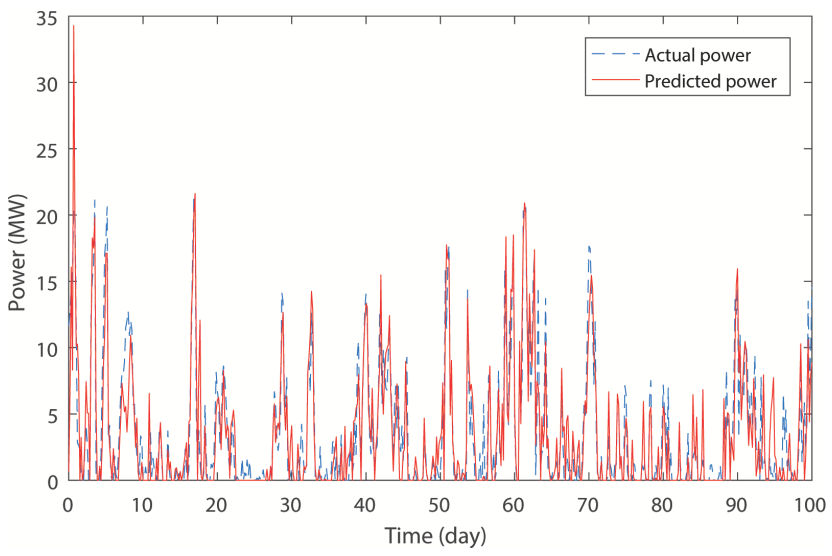

Fig. 7. Actual power $p_{t_{i}}^{*}$ and the predicted power $\hat{p}_{t_{i}}$ using a group of 10 neural networks with our best-performing system configuration (Table VIII) for a period of 100 days: (a) and (b) show the results for WF-A and WF-B, respectively. In the vast majority of cases, our system accurately predicted the production peaks.

\section{CONCLUSION}

This paper presented a novel decision support system that can predict the wind power production of a general wind farm. The system includes two main functions: long-term prediction of the power produced by a wind farm as well as computation of the related variability index and automatic 
TABLE IX

ACCURACY OF OUR SYSTEM AND A COMMERCIAL PREDICTOR APPLIED TO THE REAL ENERGY MARKET FOR A TIME SPAN OF 70 DAYS.

\begin{tabular}{ccc}
\hline \hline \multirow{2}{*}{ Wind farm } & \multicolumn{2}{c}{ WMAE } \\
& Our system & Commercial \\
\hline WF-C & 0.53 & 0.74 \\
WF-D & 0.51 & 0.52 \\
WF-E & 0.54 & 0.69 \\
WF-F & 0.55 & 0.61 \\
WF-G & 0.59 & 0.57 \\
WF-H & 0.42 & 0.57 \\
WF-I & 0.47 & 0.60 \\
WF-J & 0.58 & 0.57 \\
WF-K & 0.55 & 0.62 \\
WF-L & 0.47 & 0.53 \\
Mean & 0.52 & 0.60 \\
\hline \hline
\end{tabular}

tools that evaluate the production characteristics of wind farms and the accuracy of weather forecasts. The main novelty presented in this paper consists of our system for predicting the power production of a wind farm based on NWP data. The proposed system is able to automatically learn the production characteristics of a wind farm. The system is composed of the following modules: data harmonization, feature extraction, calibration, thresholding, and neural prediction. To achieve the best performance for heterogeneous wind farms, we proposed different configurations of the system obtained by using different combinations of its modules.

We evaluated the performance of the proposed system on data collected for two wind farms over a two-year period. Using these data, we analyzed different prediction approaches, feature sets, training strategies, system configurations, and techniques based on multiple regression strategies. The results achieved were satisfactory and demonstrated the feasibility of the proposed system. A player in the energy sector evaluated our decision support system on a set of 10 wind farms for the day-after energy market. The system was used successfully in these real-world application conditions and performed better than the system previously used by the company.

In contrast to most of the previous work in the literature, our system has an important advantage in that it can be applied to heterogeneous wind farms without requiring any previous knowledge of the plant or concerning the orography of the territory, because our system learns these types of information directly from the data. Because our system uses only NWP data, it is also applicable for wind farms from which it is difficult or impossible to obtain real-time sensor measurements. Furthermore, training the prediction system requires only approximately one hour; therefore, it is possible to periodically re-train the system in real application scenarios. Our decision support system is currently in use by a power company.

\section{REFERENCES}

[1] F. Manzano-Agugliaro, A. Alcayde, F. G. Montoya, A. Zapata-Sierra, and C. Gil, "Scientific production of renewable energies worldwide: An overview," Renewable and Sustainable Energy Reviews, vol. 18, pp. 134-143, 2013.
[2] Z. Geng, J. Chen, and Y. Han, "Energy efficiency prediction based on PCA-FRBF model: A case study of ethylene industries," IEEE Trans. on Systems, Man, and Cybernetics: Systems, vol. 47, no. 8, pp. 1763-1773, August 2017.

[3] C. M. Cheng, S. L. Tsao, and P. Y. Lin, "SEEDS: A solar-based energyefficient distributed server farm," IEEE Trans. on Systems, Man, and Cybernetics: Systems, vol. 45, no. 1, pp. 143-156, January 2015.

[4] R. Carli, M. Dotoli, R. Pellegrino, and L. Ranieri, "A decision making technique to optimize a buildings' stock energy efficiency," IEEE Trans. on Systems, Man, and Cybernetics: Systems, vol. 47, no. 5, pp. 794-807, May 2017.

[5] M. G. Simões, R. Roche, E. Kyriakides, S. Suryanarayanan, B. Blunier, K. D. McBee, P. H. Nguyen, P. F. Ribeiro, and A. Miraoui, "A comparison of smart grid technologies and progresses in europe and the u.s." IEEE Trans. on Industry Applications, vol. 48, no. 4, pp. 11541162, July 2012.

[6] M. M. B. R. Vellasco, M. A. C. Pacheco, L. S. R. Neto, and F. J. de Souza, "Electric load forecasting: evaluating the novel hierarchical neuro-fuzzy BSP model," Int. Journal of Electrical Power \& Energy Systems, vol. 26, no. 2, pp. 131-142, 2004.

[7] Z. Y. Xu, H. N. Qu, W. H. Shao, and W. S. Xu, "Virtual power plantbased pricing control for wind/thermal cooperated generation in China," IEEE Trans. on Systems, Man, and Cybernetics: Systems, vol. 46, no. 5, pp. 706-712, May 2016.

[8] Q. Yang, G. Q. Chen, S. Liao, Y. H. Zhao, H. W. Peng, and H. P. Chen, "Environmental sustainability of wind power: An emergy analysis of a Chinese wind farm," Renewable and Sustainable Energy Reviews, vol. 25, pp. 229-239, 2013.

[9] World Wind Energy Association (WWEA), "WWEA half-year report," 2016.

[10] X. K. Gu, G. F. Fan, X. R. Wang, H. X. Zhao, and H. Z. Dai, "Summarization of wind power prediction technology," Power System Technology, vol. 31, no. 2, pp. 335-338, 2007.

[11] A. Giannitrapani, S. Paoletti, A. Vicino, and D. Zarrilli, "Bidding wind energy exploiting wind speed forecasts," IEEE Trans. on Power Systems, vol. 31, no. 4, pp. 2647-2656, 2016.

[12] A. F. Taha and J. H. Panchal, "Decision-making in energy systems with multiple technologies and uncertain preferences," IEEE Trans. on Systems, Man, and Cybernetics: Systems, vol. 44, no. 7, pp. 894-907, July 2014.

[13] A. Rikalovic, I. Cosic, R. Donida Labati, and V. Piuri, "Intelligent decision support system for industrial site classification: A GIS-based hierarchical neuro-fuzzy approach," IEEE Systems Journal, 2017.

[14] S. S. Soman, H. Zareipour, O. Malik, and P. Mandal, "A review of wind power and wind speed forecasting methods with different time horizons," in Proc. of the North American Power Symp. (NAPS), September 2010, pp. $1-8$.

[15] V. Nikolić, S. Sajjadi, D. Petković, S. Shamshirband, Ž. Ćojbašić, and L. Y. Por, "Design and state of art of innovative wind turbine systems," Renewable and Sustainable Energy Reviews, vol. 61, pp. 258-265, 2016.

[16] D. Petković, Ž. Ćojbašič, and V. Nikolić, "Adaptive neuro-fuzzy approach for wind turbine power coefficient estimation," Renewable and Sustainable Energy Reviews, vol. 28, pp. 191-195, 2013.

[17] V. Nikolić, V. V. Mitić, L. Kocić, and D. Petković, "Wind speed parameters sensitivity analysis based on fractals and neuro-fuzzy selection technique," Knowledge and Information Systems, vol. 52, no. 1, pp. 255265, July 2017.

[18] D. Petković, N. T. Pavlović, and Ž. Ćojbašić, "Wind farm efficiency by adaptive neuro-fuzzy strategy," International Journal of Electrical Power \& Energy Systems, vol. 81, pp. 215-221, 2016.

[19] D. Petković, S. H. Ab Hamid, Ž. Ćojbašić, and N. T. Pavlović, "Adapting project management method and ANFIS strategy for variables selection and analyzing wind turbine wake effect," Natural Hazards, vol. 74, no. 2, pp. 463-475, November 2014.

[20] D. Petković, V. Nikolić, V. V. Mitić, and L. Kocić, "Estimation of fractal representation of wind speed fluctuation by artificial neural network with different training algorothms," Flow Measurement and Instrumentation, vol. 54 , pp. 172-176, 2017

[21] J. Zhao, Y. Guo, X. Xiao, J. Wang, D. Chi, and Z. Guo, "Multi-step wind speed and power forecasts based on a WRF simulation and an optimized association method," Applied Energy, vol. 197, pp. 183-202, 2017.

[22] J. Koo, G. D. Han, H. J. Choi, and J. H. Shim, "Wind-speed prediction and analysis based on geological and distance variables using an artificial neural network: A case study in South Korea," Energy, vol. 93, no. Part 2, pp. 1296-1302, 2015. 
[23] S. M. Lawan, W. A. W. Z. Abidin, T. Masri, W. Y. Chai, and A. Baharun, "Wind power generation via ground wind station and topographical feedforward neural network (T-FFNN) model for small-scale applications," Journal of Cleaner Production, vol. 143, pp. 1246-1259, 2017.

[24] J. Wang, Y. Song, F. Liu, and R. Hou, "Analysis and application of forecasting models in wind power integration: A review of multistep-ahead wind speed forecasting models," Renewable and Sustainable Energy Reviews, vol. 60, pp. 960-981, 2016.

[25] J. Jung and R. P. Broadwater, "Current status and future advances for wind speed and power forecasting," Renewable and Sustainable Energy Reviews, vol. 31, pp. 762-777, 2014.

[26] M. A. Mohandes, S. Rehman, and S. M. Rahman, "Estimation of wind speed profile using adaptive neuro-fuzzy inference system (ANFIS)," Applied Energy, vol. 88, no. 11, pp. 4024 - 4032, 2011.

[27] M. Lange and U. Focken, Physical approach to short-term wind power prediction. Springer, 2006.

[28] S. Salcedo-Sanz, Á. M. Pérez-Bellido, E. G. Ortiz-García, A. PortillaFigueras, L. Prieto, and D. Paredes, "Hybridizing the fifth generation mesoscale model with artificial neural networks for short-term wind speed prediction," Renewable Energy, vol. 34, no. 6, pp. 1451-1457, 2009.

[29] R. Donida Labati, A. Genovese, E. Muñoz, V. Piuri, F. Scotti, and G. Sforza, "Computational intelligence for industrial and environmental applications," in Proc. of the $8^{\text {th }}$ IEEE Int. Conf. on Intelligent Systems (IS), 2016, pp. 8-14.

[30] E. M. Petriu, G. G. Patry, T. E. Whalen, A. Al-Dhaher, and V. Z. Groza, "Intelligent robotic sensor agents for environment monitoring," in Proc. of the IEEE Int. Symp. on Virtual and Intelligent Measurement Systems (VIMS), 2002, pp. 14-19.

[31] R. Donida Labati, A. Genovese, V. Piuri, and F. Scotti, "Wildfire smoke detection using computational intelligence techniques enhanced with synthetic smoke plume generation," IEEE Trans. on Systems, Man, and Cybernetics: Systems, vol. 43, no. 4, pp. 1003-1012, July 2013.

[32] L. J. Herrera, H. Pomares, I. Rojas, A. Guillén, A. Prieto, and O. Valenzuela, "Recursive prediction for long term time series forecasting using advanced models," Neurocomputing, vol. 70, no. 1618, pp. 2870-2880, 2007.

[33] H. Dhahri and A. M. Alimi, "The modified differential evolution and the RBF (MDE-RBF) neural network for time series prediction," in Proc. of the IEEE Int. Joint Conf. on Neural Network (IJCNN), 2006, pp. 2938-2943.

[34] J. N. K. Liu, K. M. Kwong, and P. W. Chan, "Chaotic oscillatory-based neural network for wind shear and turbulence forecast with LiDAR data," IEEE Trans. on Systems, Man, and Cybernetics, Part C (Applications and Reviews), vol. 42, no. 6, pp. 1412-1423, November 2012.

[35] Z. Vale, G. K. Venayagamoorthy, J. Ferreira, and H. Morais, Computational Intelligence Applications for Future Power Systems. Dordrecht: Springer Netherlands, 2011, pp. 176-193.

[36] M. Lazzaroni, S. Ferrari, V. Piuri, A. Salman, L. Cristaldi, and M. Faifer, "Models for solar radiation prediction based on different measurement sites," Measurement, vol. 63, pp. 346-363, 2015.

[37] S. Ferrari, M. Lazzaroni, V. Piuri, A. Salman, L. Cristaldi, and M. Faifer, "Computational intelligence models for solar radiation prediction," in Proc. of the 2013 IEEE Int. Instrumentation and Measurement Technology Conf. (I2MTC), May 2013, pp. 757-762.

[38] M. Mao, J. Ling, L. Chang, N. D. Hatziargyriou, J. Zhang, and Y. Ding, "A novel short-term wind speed prediction based on MFEC," IEEE Journal of Emerging and Selected Topics in Power Electronics, vol. 4, no. 4, pp. 1206-1216, December 2016.

[39] M. B. Ozkan and P. Karagoz, "A novel wind power forecast model: Statistical hybrid wind power forecast technique (SHWIP)," IEEE Trans. on Industrial Informatics, vol. 11, no. 2, pp. 375-387, April 2015.

[40] H.-Z. Wang, G.-Q. Li, G.-B. Wang, J.-C. Peng, H. Jiang, and Y.-T. Liu, "Deep learning based ensemble approach for probabilistic wind power forecasting," Applied Energy, vol. 188, pp. 56-70, 2017.

[41] A. S. Qureshi, A. Khan, A. Zameer, and A. Usman, "Wind power prediction using deep neural network based meta regression and transfer learning," Applied Soft Computing, vol. 58, pp. 742-755, 2017.

[42] H. Liu, H. qi Tian, and Y.-F. Li, "Comparison of new hybrid FEEMDMLP, FEEMD-ANFIS, Wavelet Packet-MLP and Wavelet PacketANFIS for wind speed predictions," Energy Conversion and Management, vol. 89, pp. 1-11, 2015.

[43] H. Peng, F. Liu, and X. Yang, "A hybrid strategy of short term wind power prediction," Renewable Energy, vol. 50, pp. 590-595, 2013.

[44] A. Zameer, J. Arshad, A. Khan, and M. A. Z. Raja, "Intelligent and robust prediction of short term wind power using genetic programming based ensemble of neural networks," Energy Conversion and Management, vol. 134, pp. 361-372, 2017.

[45] R. Ata, "Artificial neural networks applications in wind energy systems: a review," Renewable and Sustainable Energy Reviews, vol. 49, pp. 534562, 2015.

[46] D. Petković, Ž. Ćojbašić, V. Nikolić, S. Shamshirband, M. L. M. Kiah, N. B. Anuar, and A. W. A. Wahab, "Adaptive neuro-fuzzy maximal power extraction of wind turbine with continuously variable transmission," Energy, vol. 64, pp. 868-874, 2014.

[47] M. S. Islam, M. A. Mohandes, and S. Rehman, "Vertical extrapolation of wind speed using artificial neural network hybrid system," Neural Computing and Applications, vol. 28, no. 8, pp. 2351-2361, August 2017.

[48] M. A. Mohandes and S. Rehman, "Short term wind speed estimation in saudi arabia," Journal of Wind Engineering and Industrial Aerodynamics, vol. 128, pp. 37-53, 2014.

[49] N. Amjady, F. Keynia, and H. Zareipour, "Wind power prediction by a new forecast engine composed of Modified Hybrid Neural Network and enhanced Particle Swarm Optimization," IEEE Trans. on Sustainable Energy, vol. 2, no. 3, pp. 265-276, July 2011.

[50] A. Tascikaraoglu and M. Uzunoglu, "A review of combined approaches for prediction of short-term wind speed and power," Renewable and Sustainable Energy Reviews, vol. 34, pp. 243-254, 2014.

[51] T. G. Barbounis, J. B. Theocharis, M. C. Alexiadis, and P. S. Dokopoulos, "Long-term wind speed and power forecasting using local recurrent neural network models," IEEE Trans. on Energy Conversion, vol. 21, no. 1, pp. 273-284, March 2006.

[52] D. A. Fadare, "The application of artificial neural networks to mapping of wind speed profile for energy application in Nigeria," Applied Energy, vol. 87, no. 3, pp. 934-942, 2010.

[53] C. Y. Zhang, C. L. P. Chen, M. Gan, and L. Chen, "Predictive Deep Boltzmann Machine for multiperiod wind speed forecasting," IEEE Trans. on Sustainable Energy, vol. 6, no. 4, pp. 1416-1425, October 2015.

[54] K. Philippopoulos and D. Deligiorgi, "Application of artificial neural networks for the spatial estimation of wind speed in a coastal region with complex topography," Renewable Energy, vol. 38, no. 1, pp. 7582, 2012.

[55] Z. O. Olaofe, "A 5-day wind speed \& power forecasts using a layer recurrent neural network (LRNN)," Sustainable Energy Technologies and Assessments, vol. 6, pp. 1-24, 2014.

[56] G. Sideratos and N. D. Hatziargyriou, "Probabilistic wind power forecasting using Radial Basis Function Neural Networks," IEEE Trans. on Power Systems, vol. 27, no. 4, pp. 1788-1796, November 2012

[57] G. Sideratos and N. D. Hatziargyriou, "An advanced statistical method for wind power forecasting," IEEE Trans. on Power Systems, vol. 22, no. 1, pp. 258-265, February 2007.

[58] S. Buhan, Y. Özkazanç, and I. Cadırcı, "Wind pattern recognition and reference wind mast data correlations with NWP for improved windelectric power forecasts," IEEE Trans. on Industrial Informatics, vol. 12, no. 3, pp. 991-1004, June 2016.

[59] L. Zjavka, "Wind speed forecast correction models using polynomial neural networks," Renewable Energy, vol. 83, pp. 998-1006, 2015.

[60] A. Agrawal and K. S. Sandhu, "Most influential parametrical and data needs for realistic wind speed prediction," Renewable Energy, vol. 94, pp. $452-465,2016$.

[61] S. Salcedo-Sanz, Á. M. Pérez-Bellido, E. G. Ortiz-García, A. PortillaFigueras, L. Prieto, and F. Correoso, "Accurate short-term wind speed prediction by exploiting diversity in input data using banks of artificial neural networks," Neurocomputing, vol. 72, no. 4, pp. 1336-1341, 2009.

[62] I. J. Ramirez-Rosado, L. A. Fernandez-Jimenez, C. Monteiro, J. Sousa, and R. Bessa, "Comparison of two new short-term wind-power forecasting systems," Renewable Energy, vol. 34, no. 7, pp. 1848-1854, 2009.

[63] J. C. Palomares-Salas, A. Agüera-Pérez, J. J. G. de la Rosa, and A. Moreno-Muñoz, "A novel neural network method for wind speed forecasting using exogenous measurements from agriculture stations," Measurement, vol. 55, pp. 295-304, 2014.

[64] PSU/NCAR, "MM5 modeling system overview." [Online]. Available: http://www2.mmm.ucar.edu/mm5/overview.html

[65] WRF, "The Weather Research \& Forecasting Model." [Online]. Available: http://www.wrf-model.org/index.php

[66] CNRM, "Aire Limitée Adaptation dynamique Développement InterNational (ALADIN)." [Online]. Available: http://www.umr-cnrm. fr/aladin-old/

[67] L. Dong, L. Wang, S. F. Khahro, S. Gao, and X. Liao, "Wind power day-ahead prediction with cluster analysis of NWP," Renewable and Sustainable Energy Reviews, vol. 60, pp. 1206-1212, 2016. 
[68] A. K. Jain, P. Flynn, and A. A. Ross, Handbook of Biometrics. Secaucus, NJ, USA: Springer-Verlag New York, Inc., 2007.

[69] R. O. Duda, P. E. Hart, and D. G. Stork, Pattern classification, $2^{\text {nd }}$ ed. John Wiley \& Sons, 2000.

[70] C. Bergmeir and J. M. Benítez, "On the use of cross-validation for time series predictor evaluation," Information Sciences, vol. 191, pp. 192213, 2012.

[71] H. Madsen, P. Pinson, G. Kariniotakis, H. A. Nielsen, and T. S. Nielsen, "Standardizing the performance evaluation of short-term wind power prediction models," Wind Engineering, vol. 29, no. 6, pp. 475-489, 2005.

[72] D. M. Quan, E. Ogliari, F. Grimaccia, S. Leva, and M. Mussetta, "Hybrid model for hourly forecast of photovoltaic and wind power," in Proc. of the 2013 IEEE Int. Conf. on Fuzzy Systems (FUZZ-IEEE), 2013, pp. $1-6$.

[73] J. Zack, "Overview of wind energy generation forecasting," TrueWind Solutions, LLC. \& AWS Scientific, Inc, Tech. Rep., 2003.

[74] C. J. Willmott and K. Matsuura, "Advantages of the mean absolute error (MAE) over the root mean square error (RMSE) in assessing average model performance," Climate Research, vol. 30, no. 1, pp. 79-82, 2005.

[75] E. Zivot and J. Wang, Eds., Vector Autoregressive Models for Multivariate Time Series. Springer New York, 2006, pp. 385-429.

[76] I. Guyon and A. Elisseeff, "An introduction to variable and feature selection," The Journal of Machine Learning Research, vol. 3, pp. 11571182, March 2003.

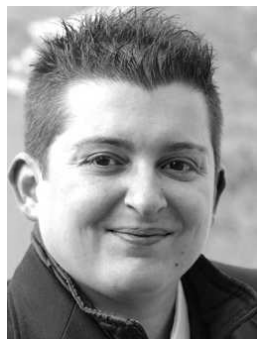

Ruggero Donida Labati has received his Ph.D. in computer science at Università degli Studi di Milano, Italy (2013). He is Assistant Professor in computer science at the Università degli Studi di Milano, Italy (since 2015). He has been Visiting Researcher at the Michigan State University, MI, USA. His main research interests are: intelligent systems, signal and image processing, machine learning, pattern analysis and recognition, theory and industrial applications of neural networks, biometrics, and industrial applications. Original results have been published in more than 50 papers in international journals, proceedings of international conferences, books, and book chapters. He is Associate Editor of the Springer Journal of Ambient Intelligence and Humanized Computing.

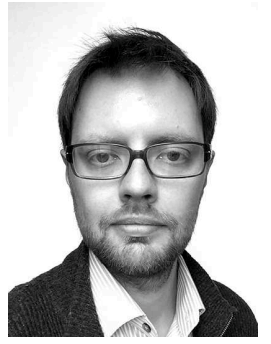

Angelo Genovese has received his Ph.D. in computer science at Università degli Studi di Milano, Italy (2014). He is Postodoctoral Research Fellow in computer science at the Università degli Studi di Milano, Italy (since 2014). He has been a visiting researcher at the University of Toronto, ON, Canada. His main research interests are: signal and image processing, three-dimensional reconstruction, computational intelligence technologies for biometric systems, industrial and environmental monitoring systems, and design methodologies and algorithms for self-adapting systems. Original results have been published in more than 30 papers in international journals, proceedings of international conferences, books, and book chapters. He is Associate Editor of the Springer Journal of Ambient Intelligence and Humanized Computing.

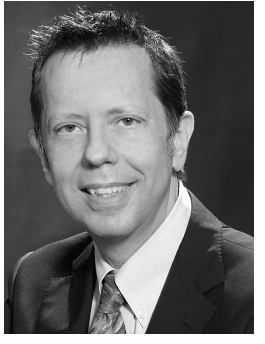

Vincenzo Piuri has received his Ph.D. in computer engineering at Politecnico di Milano, Italy (1989). $\mathrm{He}$ is Full Professor in computer engineering at the Università degli Studi di Milano, Italy (since 2000). $\mathrm{He}$ has been Associate Professor at Politecnico di Milano, Italy and Visiting Professor at the University of Texas at Austin and at George Mason University, USA. His main research interests are: intelligent systems, signal and image processing, machine learning, pattern analysis and recognition, theory and industrial applications of neural networks, biometrics, intelligent measurement systems, industrial applications, fault tolerance, digital processing architectures, and cloud computing infrastructures. Original results have been published in more than 350 papers in international journals, proceedings of international conferences, books, book chapters, and patents. $\mathrm{He}$ is Fellow of the IEEE, Distinguished Scientist of ACM, and Senior Member of INNS. He has been IEEE Vice President for Technical Activities (2015), IEEE Director, President of the IEEE Computational Intelligence Society, Vice President for Education of the IEEE Biometrics Council, Vice President for Publications of the IEEE Instrumentation and Measurement Society and the IEEE Systems Council, and Vice President for Membership of the IEEE Computational Intelligence Society. He is Editor-in-Chief of the IEEE Systems Journal (2013-19), and Associate Editor of the IEEE Transactions on Computers, the IEEE Transactions on Cloud Computing, and IEEE Access, and has been Associate Editor of the IEEE Transactions on Neural Networks and the IEEE Transactions on Instrumentation and Measurement. $\mathrm{He}$ received the IEEE Instrumentation and Measurement Society Technical Award (2002). He is Honorary Professor at Obuda University, Budapest, Hungary, Guangdong University of Petrochemical Technology, China, Muroran Institute of Technology, Japan, and the Amity University, India.

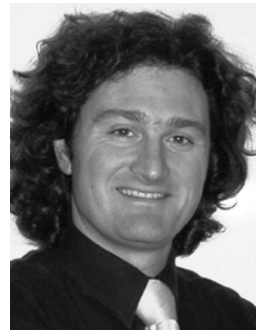

Fabio Scotti has received his Ph.D. in computer engineering at Politecnico di Milano, Italy (2003). $\mathrm{He}$ is Associate Professor in computer science at the Università degli Studi di Milano, Italy (since 2015). His main research interests are: biometric systems, machine learning and computational intelligence, signal and image processing, theory and applications of neural networks, three-dimensional reconstruction, industrial applications, intelligent measurement systems, and high-level system design. Original results have been published in more than 100 papers in international journals, proceedings of international conferences, books, book chapters, and patents. He is IEEE Senior Member. He is Associate Editor of the IEEE Transactions on Human-Machine Systems and the Springer Soft Computing, he has been Associate Editor of the IEEE Transactions on Information Forensics and Security, and Guest co-editor for the IEEE Transactions on Instrumentation and Measurement.

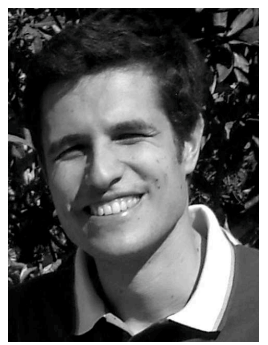

Gianluca Sforza has received his Ph.D. in computer science at Università degli Studi di Bari, Italy (2012). He is Postdoctoral Research Fellow in computer science at the Istituto Nazionale di Fisica Nucleare, Bari Section, Italy (since 2017). He has been visiting student at the Missouri University of Science and Technology, Rolla, MO, USA, and visiting scholar at the University of Reading, UK. His main research interests are: image analysis, visual perception, recursion theory, machine learning, biometrics, and industrial applications. Original results have been published in more than 20 papers in international journals and proceedings of international conferences. 